\title{
Charged particles in higher dimensional homogeneous gravitational field: self-energy and self-force
}

\author{
Valeri P. Frolov and Andrei Zelnikov \\ Theoretical Physics Institute, Department of Physics, University of Alberta, \\ Edmonton, AB, T6G 2E1 Canada \\ E-mail: vfrolov@ualberta.ca, zelnikov@ualberta.ca
}

ABstract: A problem of self-energy and self-force for a charged point-like particle in a higher dimensional homogeneous gravitational field is considered. We study two cases, when a particle has the usual electric charge, and when it has a scalar charge, which is a source of a scalar massless minimally coupled field. We assume that a particle is at rest in the gravitational field, so that its motion is not geodesic, and it has an acceleration $a$ directed away from the horizon. The self-energy of a point charge is divergent and the strength of the divergence grows with the number of dimensions. In order to obtain a finite contribution to the self-energy, we use a covariant regularization method which is a modification of the proper time cut-off and other covariant regularizations. We analyze the relation between the self-energy and the self-force and obtain explicit expressions for the self-forces for the electric and the scalar charge in spacetimes with the number of dimensions up to eight. General expressions for the case of higher dimensions are also obtained. We discuss special logarithmic factors $\ln a$, which are present in both the self-energy and the self-force in odd dimensions. Finally, we compare the obtained results with the earlier known results both for the homogeneous gravitational field and for particles near black holes.

Keywords: Field Theories in Higher Dimensions, Black Holes, Renormalization Regularization and Renormalons

ARXIV EPRINT: 1407.3323 


\section{Contents}

1 Introduction 1

2 An electric charge in a static spacetime 5

2.1 Equations 5

$\begin{array}{lll}2.2 \text { Energy } & 7\end{array}$

2.3 Self-force 9

3 Electric charge in the Rindler spacetime $\quad 10$

$\begin{array}{lll}3.1 & \text { Static Green function } & 10\end{array}$

$\begin{array}{lll}3.2 & \text { Near-horizon limit of the Schwarzschild black hole } & 14\end{array}$

4 Electromagnetic self-energy and self-force: results $\quad 15$

$\begin{array}{ll}4.1 \text { Divergent part of the static Green function } & 15\end{array}$

$\begin{array}{llr}4.2 \text { Self-force } & 16\end{array}$

$\begin{array}{llr}4.3 & \text { Self-energy } & 18\end{array}$

$\begin{array}{lll}4.4 \text { Summary of results } & 19\end{array}$

5 A scalar charge in a static spacetime 20

$\begin{array}{lll}5.1 \text { Equations } & 20\end{array}$

5.2 Self-energy 21

$\begin{array}{lll}5.3 & \text { Self-force } & 21\end{array}$

6 Scalar charge in the Rindler spacetime $\quad 23$

7 Scalar self-energy and self-force: results $\quad \mathbf{2 5}$

$\begin{array}{lll}7.1 & \text { Divergent part of the static Green function } & 25\end{array}$

$\begin{array}{lll}7.2 & \text { Self-force } & 25\end{array}$

$\begin{array}{lll}7.3 & \text { Self-energy } & 27\end{array}$

$\begin{array}{ll}7.4 & \text { Summary of results }\end{array}$

$\begin{array}{llr}8 & \text { Discussion } & 28\end{array}$

A Motion of a continuous charged medium in a curved spacetime $\quad 30$

$\begin{array}{lll}\text { A.1 Fock's approach } & 30\end{array}$

A.2 Electrically charged media 32

A.2.1 Equations of motion 32

$\begin{array}{ll}\text { A.2.2 Stress-energy tensor } & 33\end{array}$

A.3 Motion of the media with the scalar charge 33

A.3.1 Equations of motion 33

$\begin{array}{lll}\text { A.3.2 Stress-energy tensor } & 34\end{array}$

B Green Functions $\quad 34$ 


\section{Introduction}

There are several 'eternal problems' in the theoretical physics, that have been discussed already for several decades. The problem of the electromagnetic mass of an electron is one of them. A charge is a source of the electromagnetic field. The latter has energy and, when a charged particle moves, it has momentum as well. The energy of the field contributes to the total energy of the particle, and at least a part of its proper mass is connected with this contribution. In the classical physics an electron is a point particle, so that its electromagnetic energy diverges. In the simplest case when the charge $e$ is distributed uniformly over the surface of a sphere of the radius $\varepsilon$ the electromagnetic contribution to the self energy is

$$
E=e^{2} /(2 \varepsilon) .
$$

The electromagnetic field of a charged particle is distributed in space. Its configuration, and hence its energy, depends on the boundary conditions. Hence, in a general case the field contribution to the self-energy besides the local divergent part contains an additional term which depends on properties of the matter outside the charge, as well as the boundary conditions. In a general case, the latter is non-local. If such a contribution depends on the position of the charge and changes when the charge changes its location, then there exists a non-local self-force acting on the charge.

Similar effect exists when a charge is located in a spacetime with non-trivial gravitational field, for example, near a black hole. The black hole metric acts on the field generated by a charge, in a way, similar to a dielectric [1] with the inhomogeneous refraction index. It deforms the electric field so that it is not only decreasing at infinity, but takes very special form at the horizon of the black hole, which, for example, guarantees the regularity of the field in a freely falling frame. However, using the analogy with a dielectric, it can be quite tricky to arrive to an intuitive explanation of a correct scaling of the self-force on the distance from the horizon and even a sign of the effect (see discussion in [2]). Concrete calculations are often required to get the correct answer. Knowledge of exact solutions in some cases would be of great luck and help.

When the mass of the black hole is large, the curvature near the event horizon is small. The horizon becomes practically flat and the gravitational field near it is approximately static and homogeneous. In other words, the geometry in the vicinity of the horizon can be approximated by a Rindler metric. If a charge is located close to the horizon the field near it is similar to the field of a charge in the Rindler space. However, at far distances from the charge the difference in the boundary conditions and the topology of the horizon for these two cases (the charge near the black hole and in the Rindler spacetime) becomes 
important for the self-energy problem. We shall discuss this difference in the present paper and demonstrate that it is related to the contributions of zero-modes of the corresponding elliptic field operator.

First calculation of the electromagnetic self-energy of a charge in a static homogeneous gravitational field was performed by Fermi in 1921 [3]. In particular, he demonstrated that the 'weight' of a system of electric charges is a product of its electrostatic energy by the gravitational acceleration, so that the electromagnetic contribution to the gravitational mass is identical to (properly calculated) contribution to its inertial mass, as it is required by the equivalence principle.

For a charge located near a static vacuum black hole the self-energy was calculated in [4]. In the case of an electric charge near a four-dimensional Schwarzschild black hole the self-force is repulsive. Later this result was generalized to the case of electric and scalar charges near Reissner-Nordström black holes $[5,6]$ and stationary, axisymmetric black holes [7].

Expressions for the finite part of the self-energy in the homogeneous gravitational field can be obtained by straightforward calculations [6]. The result is very simple

$$
E^{\mathrm{res}}=-\frac{1}{2} e^{2} a .
$$

The purpose of this paper is to generalize these results to the case when a charged particle is in a homogeneous gravitational field in the higher dimensional spacetime. There are several reasons why this problem might be interesting. First of all, higher dimensional aspects of the high energy physics attracted a lot of interest in connection with different models of the brane worlds and large extra dimensions. In such models the fundamental scale of the quantum gravity can be in the energy range of several $\mathrm{TeV}$, which opens an intriguing possibility of the micro black hole creation in modern colliders. An interesting question is how the problem of the self-energy is modified when the spacetime has one or more extra dimensions. Another reason that makes this problem interesting is purely theoretical curiosity. The electromagnetic field in the higher dimensional spacetime is not conformal invariant. Moreover, in the case when the spacetime has odd number of dimensions, the Huygens-Fresnel principle is violated even in flat spacetime, namely, the retarded Green function is not localized on the surface of the past null cones, but has 'tail' inside it. Static Green functions, that are the main topic of this paper, also reveal specific properties that are alternating with the odd-even dimensionatity of the spacetime. Recently, Beach et al. [2] calculated the self-energy and self-force for an electric and scalar charges near 5dimensional spherically symmetric vacuum black hole. They explicitly demonstrates the presence of a special logarithmic factor in the expression for the self-force.

An interesting consequence of this phenomena is the following. It can be shown that the self-energy problem for a point charge in a static $D$-dimensional spacetime can be reduced to the calculation of the quantum fluctuations of a scalar field in the Euclidean $(D-1)$ dimensional theory (see [8]). When $D$ is odd, the corresponding $(D-1)$-theory possesses quantum anomalies. Their contribution to the self-energy of a charge in a MajumdarPapapetrou class of metrics was analyzed in $[9,10]$. In three dimensions this anomaly was used to calculate the self-energy of dipoles [11]. 
In the present paper we focus on a simpler problem: the self-energy and the self-force of a point charge in the static homogeneous higher dimensional gravitational field. We assume that a charge is at rest in such a field. Since its worldline is not geodesic, it has acceleration, which we denote by $a$. Let us emphasize that according to the equivalence principle, this problem is identical to a study of the self-force acting on a uniformly accelerated charge in the Minkowski spacetime in the absence of the gravitational field.

Because, besides the charge $e$, this problem contains only one dimensional parameter, the value of the acceleration, an expected expression for the self-energy and the self-force can be easily obtained up to a numerical factor by simple arguments based on the dimensional analysis. First of all, let us notice that the dimensionality of the electric charge depends on the number of dimensions. For example, the energy $E$ of the interaction of two equal charges $e$, and the force $f$ between them in $D$-dimensional spacetime have the form

$$
E \sim \frac{e^{2}}{R^{D-3}}, \quad f \sim \frac{e^{2}}{R^{D-2}} .
$$

Here $R$ is the distance between the charges.

As we shall see, for a point particle both self-energy and self-force are divergent. Based on relations (1.3) one may arrive to a correct result that the leading divergence of these quantities is

$$
E^{\text {div }} \sim \frac{e^{2}}{\epsilon^{D-3}}, \quad f^{\text {div }} \sim \frac{e^{2}}{\epsilon^{D-3}} a .
$$

One can define a finite residual part of the self-energy and the self-force, which are obtained by subtraction of all the divergences. Both residual self-energy and self-force are also proportional to the charge squared, while $a^{-1}$ has the dimensionality of the length. ${ }^{1}$ Thus one can write for them the expressions

$$
E^{\mathrm{res}} \sim e^{2} a^{D-3}, \quad f^{\mathrm{res}} \sim e^{2} a^{D-2} .
$$

It is easy to see that in the four-dimensional case the expression for $E^{\text {res }}$ correctly reproduces (up to a numerical factor) the exact result eq. (1.2). In the five-dimensional case, the corresponding exact expressions can be obtained by taking proper limit of the results of [2]. By the comparison of the exact results [2] with eq. (1.5) one finds out that in the five dimensions these expressions should be modified by a logarithmic term. Our calculations in the present paper confirm that such a logarithmic factor appears in all odd-dimensional cases

$$
E^{\text {res }} \sim e^{2} a^{D-3} \ln (a l), \quad f^{\text {res }} \sim e^{2} a^{D-2} \ln (a l), \quad D \text { is odd } .
$$

The parameter $l$ which is required in order to make the expression under the logarithm dimensionless, is an infrared (IR) cut-off. It should be emphasized that the case of the homogeneous gravitational field is not realistic and is a certain idealization. The gravitational field created by an extended compact object can be approximated by the homogeneous one only in a domain, where its spatial change (and hence curvature) can be neglected. Similarly, one can support uniform acceleration only for some finite interval

\footnotetext{
${ }^{1}$ We use units where $G=c=1$.
} 
of time. One can expect that the parameter $l$ reflects the role of these natural infrared cut-offs.

In order to perform calculations of the self-energy for a point charge one needs at first regularize its infinities. In higher dimensions this problem becomes more severe, because divergences are stronger and have a more complicated structures. In D dimensions, when $D \geq 4$, the leading ultraviolet (UV) divergence of the self-energy is of the form $e^{2} / \epsilon^{D-3}$, where $\epsilon$ is the UV cut-off length parameter. These divergencies can be absorbed into the renormalization of the local mass of the particle. In four dimensions this mass renormalization is sufficient to make equations of motion well defined and to determine a remaining finite self-energy of the charged particle, which is the difference of energies of a charged and a neutral particles of the same renormalized masses. In higher dimensions new subleading UV divergencies appear which require special treatment. In a general case these divergences contain the acceleration and its higher derivatives as well as the curvature and its derivative (in a case of a curved spacetime) terms (see, e.g., discussion of the in $[12,13]$ ). Simple dimensional analysis shows that in our case of the homogeneous gravitational field (constant acceleration) UV-divergent terms in the self-force have the following structure

$$
f_{\mu}^{\mathrm{div}}=e^{2} a_{\mu} P, \quad P=\sum_{p=0}^{D-3} c_{D}^{(p)} \frac{a^{p}}{\epsilon^{D-3-p}} .
$$

In the odd number of spacetime dimensions $D$ the terms in the sum contain also $\ln (a \epsilon)$ contribution.

One of possible ways to deal with these divergences is to add corresponding counterterms to the action for the particle motion and choose them so that the divergences can be absorbed by a redefinition of the coefficients of these counter-terms. These counterterms are necessary to consider even in flat spacetime and even for a constant acceleration motion [14]. In six-dimensional curved spacetimes the counterterms were explicitly calculated in [13].

For a motion of a particle with the constant acceleration $a$ it is sufficient to consider only counter-terms of the form

$$
S^{\text {counter }}=-\frac{1}{2} \int Q(a) \mathbf{u}^{2} d \tau, \quad Q(a)=\sum_{p=0}^{D-3} C_{D}^{(p)} a^{p} .
$$

Here $\mathbf{u}$ is the velocity of the particle, $\tau$ is the proper time, and $Q(a)$ is a polynomial of the power $D-4$ of the acceleration $a$ (or, in odd dimensions, a function which is obtained from such a polynomial by including the $\ln (a \epsilon)$ term).

The variation of this action restricted to the motion with constant $a$ is

$$
\frac{\delta S^{\text {counter }}}{\delta x^{\mu}}=a^{\mu}\left[Q(a)-a \frac{\partial Q(a)}{\partial a}\right]
$$

Here the polynomial in the square brackets is of the same order as $Q(a)$. The chosen structure of $S^{\text {counter }}$ allows one to include the divergences eq. (1.7) into it by simple redefinition of the coefficients $C_{D}^{(p)}$ in $Q(a)$. Note that taking into account logarithmic terms is manageble and does not complicate the problem. Let us emphasize that such an approach 
would inevitably result in a theory containing higher than second derivatives in the particle equation of motion. The consistency of such a theory is a complicated problem that we are not able to discuss here. Let us mention only that if one prefers not to introduce the counter-terms similar to eq. (1.8), one may try to include the divergencies similar to eq. (1.7) into a redefinition of the proper mass of the particle. However in such a case the self-force of higher-dimensional classical point charges would become dependent on the composition of extended classical charges. In other words the self-force can be used as the probe of an internal structure of extended sources [15]. In the present paper we adopt the renormalization approach. We shall use a modification of the proper time cut-off regularization for calculation of the divergencies eq. (1.7) and calculate the finite residual part of the self-force by subtracting these divergences.

Before describing the structure of the present paper, let us make one more general remark. The problem of self-force becomes very popular in connection to the discussion of motion of compact massive objects (for example small size black holes) near a large black hole. The methods based on the general theory developed by DeWitt and Brehme [16] and their recent modifications are widely used for this purpose. We would like to remark that the non-local forces, similar to that we discuss in this paper, cannot be found in such calculations. $^{2}$

The paper is organized as follows: in the section 2 a brief review is given of the general method which we use to compute the self-energy and self-force for a point electric charge in a static spacetime. In the section 3 we apply this approach and a regularization technique to an electric charge in a homogeneous gravitational field. In the section 4 the results of calculations of the self-energy and the self-force of the electric charges in higherdimensional spacetimes are collected together. In sections 5, 6, and 7 we repeat the same steps of calculations of the self-energy and the self-force, but in application to the scalar charges. The section 8 is the summary of the results. In appendix A we provide definitions and a general derivation of the density of the self-force and self-energy for electrically and scalar charged media. In appendix B we collected the details of calculations of the Green functions expansions, used in the derivation of our final results for the self-energy and self-force.

\section{An electric charge in a static spacetime}

\subsection{Equations}

The action for the Maxwell field in a $D$-dimensional spacetime has the form

$$
I=-\frac{1}{16 \pi} \int d X \sqrt{-g^{\mathrm{D}}} F^{\mu \nu} F_{\mu \nu}+\int d X \sqrt{-g^{\mathrm{D}}} A_{\mu} J^{\mu} .
$$

Its variation with respect to the potential $A_{\mu}$ gives the field equation

$$
F_{; \nu}^{\mu \nu}=4 \pi J^{\mu}
$$

where $J^{\mu}$ is the current.

\footnotetext{
${ }^{2}$ Just to give a simple explanation, let us consider an electric charge in a flat space in the presence of a conducting mirror. Since the curvature identically vanishes in the vicinity of the charge along all its world-line, all the terms in the Brehme-DeWitt decomposition are the same as in the empty space. So that the interaction of the charge with its 'image' is beyond their approximation.
} 
Let us consider a static spacetime and write its metric in the form

$$
d s^{2}=g_{\mu \nu}^{\mathrm{D}} d X^{\mu} d X^{\nu}=-\alpha^{2} d t^{2}+g_{a b} d x^{a} d x^{b}, \quad \partial_{t} \alpha=\partial_{t} g_{a b}=0,
$$

so that one has

$$
\begin{aligned}
& X^{\mu}=\left(t, x^{a}\right), a, b=1, \ldots, D-1, \\
& g^{\mathrm{D}}=\operatorname{det} g_{\mu \nu}^{\mathrm{D}}=-\alpha^{2} g, \quad g=\operatorname{det} g_{a b}, \quad g_{00}^{\mathrm{D}}=-\alpha^{2}, \quad g_{a b}^{\mathrm{D}}=g_{a b} .
\end{aligned}
$$

This spacetime has the Killing vector $\xi^{\mu}$ describing the symmetry of the metric under the time translations. The Killing vector is defined up to an arbitrary constant factor, which can be chosen by fixing the norm of the Killing vector $\xi=\sqrt{-\xi^{\mu} \xi_{\mu}}=\alpha(x)$ to be equal to one at some point $x_{\mathrm{o}}$, that is $\alpha\left(x_{\mathrm{o}}\right)=1$. The proper time of an observer at this point coincides with the coordinate time $t$.

Later on we consider the energy of a static point charge located at some point $y$, which does not necessarily coincide with the point $x_{o}$ of the normalization of the Killing vector $\xi^{\mu}$. At the same time we shall use this vector to define the energy of the system. As a result a so calculated energy $\mathcal{E}$ is in fact a function of two variables, $\mathcal{E}\left(x_{o}, y\right)$. Similarly one obtains the regularized value and finite residual self-energy $\mathcal{E}_{\epsilon}\left(x_{o}, y\right)$ and $\mathcal{E}^{\text {res }}\left(x_{o}, y\right)$, respectively. These quantities will be useful for the calculations of the self-force, because one is able to find the change of the energy of the charge when its position $y$ changes, while the normalization point $x_{o}$ is fixed. On the other hand, when one discusses the energy of the charged particle, it is always possible to choose a position of the normalization point $x_{o}$ to coincide with the position of the charge, $y$, so that one can write

$$
E(y)=\mathcal{E}(y, y), \quad E_{\epsilon}(y)=\mathcal{E}_{\epsilon}(y, y), \quad E^{\mathrm{res}}(y)=\mathcal{E}^{\mathrm{res}}(y, y)
$$

These are the 'energies' evaluated by the observer placed at the position of the charge.

For a static source $J^{\mu}=\delta_{0}^{\mu} J^{0}$ the vector potential $A_{\mu}$ can be reduced to the only nontrivial component $A_{0}$, which obeys the equation

$$
\frac{1}{\alpha \sqrt{g}} \partial_{a}\left(\frac{1}{\alpha} \sqrt{g} g^{a b} \partial_{b} A_{0}\right)=4 \pi J^{0}
$$

Following the paper [10] we introduce another field variable $\psi$ instead of the electric potential

$$
A_{0}=-\alpha^{1 / 2} \psi
$$

Then we can rewrite our problem as that for the scalar field $\psi$ in $(D-1)$-dimensional space and interacting with the external dilaton field $\alpha$. The equation for the field $\psi$ is

$$
(\triangle+V) \psi=-4 \pi j
$$

Here,

$$
\triangle=g^{a b} \nabla_{a} \nabla_{b}
$$


is the $(D-1)$-dimensional covariant Laplace operator, $V$ is the potential, and $j$ is the effective charge density

$$
V=-\frac{3}{4} \frac{(\nabla \alpha)^{2}}{\alpha^{2}}+\frac{\triangle \alpha}{2 \alpha} \equiv-\alpha^{1 / 2} \triangle\left(\alpha^{-1 / 2}\right), \quad j \equiv \alpha^{3 / 2} J^{0} .
$$

The field $\psi$ is chosen in such a way that the operator $\mathcal{O}=(\triangle+V)$ is self-adjoint in the space with the metric $g_{a b}$.

\subsection{Energy}

We define the static Green function $G_{00}\left(x, x^{\prime}\right)$ as a solution of the equation

$$
\frac{1}{\sqrt{g}} \partial_{a}\left(\frac{1}{\alpha} \sqrt{g} g^{a b} \partial_{b} G_{00}\left(x, x^{\prime}\right)\right)=\frac{1}{\sqrt{g}} \delta\left(x-x^{\prime}\right),
$$

satisfying the properly chosen regularity conditions at the infinity and at the horizon (if the latter is present). It is easy to check that the static Green function $G_{00}\left(x, x^{\prime}\right)$ is the time integral of the $D$-dimensional retarded Green function over the whole time $t$ range. Using the static Green function one can write the vector potential in the form

$$
A_{0}(x)=4 \pi \int_{\Sigma} d x^{\prime} \alpha\left(x^{\prime}\right) \sqrt{g\left(x^{\prime}\right)} G_{00}\left(x, x^{\prime}\right) J^{0}\left(x^{\prime}\right) .
$$

The energy function $\mathcal{E}$ of a static charge distribution reads

$$
\mathcal{E}=-\int_{\Sigma} T_{\mu}^{\nu} \xi^{\mu} d \Sigma_{\nu}=-\int_{\Sigma} d x \sqrt{g} \alpha T_{0}^{0}
$$

Substituting here the electromagnetic contribution to the stress-energy tensor eq. (A.29) one can show that

$$
\mathcal{E}=\int_{\Sigma} d x \sqrt{g} \alpha^{-1} g^{a b} \partial_{a} A_{0} \partial_{b} A_{0}
$$

Taking into account the Maxwell equations and boundary conditions at infinity and at the horizon we get $[5,6,10]$

$$
\mathcal{E}=-2 \pi \int d x d x^{\prime} \sqrt{-g^{\mathrm{D}}(x)} \sqrt{-g^{\mathrm{D}}\left(x^{\prime}\right)} J^{0}(x) G_{00}\left(x, x^{\prime}\right) J^{0}\left(x^{\prime}\right) .
$$

Using the potential $\psi$ given by eq. (2.8) the energy function eq. (2.16) can be presented in the form

$$
\mathcal{E}=\frac{1}{8 \pi} \int d x \sqrt{g} g^{a b}\left(\psi_{, a}+\frac{\alpha_{, a}}{2 \alpha} \psi\right)\left(\psi_{, b}+\frac{\alpha_{, b}}{2 \alpha} \psi\right)
$$

One can write

$$
\mathcal{E}=2 \pi \int d x d x^{\prime} \sqrt{g(x)} \sqrt{g\left(x^{\prime}\right)} j(x) G\left(x, x^{\prime}\right) j\left(x^{\prime}\right) .
$$

Here $G$ is the Green function corresponding to the operator $\mathcal{O}=\triangle+V$ in $(D-1)$-dimensional space

$$
(\triangle+V) G\left(x, x^{\prime}\right)=-\delta\left(x, x^{\prime}\right)
$$


The Green functions $G$ and $G_{00}$ are related to each other as follows

$$
G_{00}\left(x, x^{\prime}\right)=-\alpha^{1 / 2}(x) \alpha^{1 / 2}\left(x^{\prime}\right) G\left(x, x^{\prime}\right) .
$$

A point electric charge $e$ moving along the worldline $\gamma$ defined by the equation $X^{\mu}=$ $Y^{\mu}(\tau)$ is described by the distribution (see, e.g., [17-19])

$$
J^{\alpha}(X)=e \int_{\gamma} d \tau g_{\mu}^{\mathrm{D} \alpha}(X, Y(\tau)) u^{\mu} \delta^{\mathrm{D}}(X, Y(\tau)) .
$$

Here $\tau$ is the proper time of the particle, $u^{\mu}=d Y^{\mu}(\tau) / d \tau$ is the velocity of the particle, $g^{\mathrm{D} \alpha}{ }_{\mu}\left(X, X^{\prime}\right)$ is the parallel transport operator, and $\delta^{\mathrm{D}}\left(X, X^{\prime}\right)$ is the invariant D-dimensional $\delta$-function

$$
\delta^{\mathrm{D}}\left(X, X^{\prime}\right)=\frac{1}{\sqrt{-g^{\mathrm{D}}}} \delta^{\mathrm{D}}\left(X-X^{\prime}\right) .
$$

We are studying static charges in the static spacetimes eq. (2.3). For a static point charge located at a fixed point $y$, the only non-vanishing component of the current is

$$
J^{0}(x)=e \frac{1}{\alpha(y) \sqrt{g(y)}} \delta(x-y) .
$$

The rescaled point current eq. (2.11) takes the form ${ }^{3}$

$$
j(x)=e \frac{\alpha^{1 / 2}(y)}{\sqrt{g(y)}} \delta(x-y) .
$$

Finally we arrive at the following relation

$$
\mathcal{E}=2 \pi e^{2} \alpha(y) G(y, y) .
$$

It was demonstrated in [10] that this relation allows the following 'elegant' interpretation. Consider eq. (2.17) as an action for a quantum field $\psi$ in a curved $D-1$ dimensional

\footnotetext{
${ }^{3}$ Charges are normalized in such a way that the interaction energy of two point charges $e_{1}$ and $e_{2}$ placed at a distance $r$ from each other in $D$-dimensional Minkowski spacetime is

$$
E=\frac{\Gamma\left(\frac{D-3}{2}\right)}{\pi^{\frac{D-3}{2}}} \cdot \frac{e_{1} e_{2}}{r^{D-3}}=\frac{4 \pi}{(D-3) \Omega_{(\mathrm{D}-2)}} \cdot \frac{e_{1} e_{2}}{r^{D-3}}, \quad \Omega_{n}=\frac{2 \pi^{(n+1) / 2}}{\Gamma((n+1) / 2)},
$$

where $\Omega_{n}$ is the area of $n$-dimensional unit sphere. The interaction force between charges in $D$ dimensions reads$$
f=\frac{4 \pi}{\Omega_{(\mathrm{D}-2)}} \cdot \frac{e_{1} e_{2}}{r^{D-3}} .
$$

In the paper [2] authors work in a different system of units, such that the force between two charges $\tilde{e}_{1}$ and $\tilde{e}_{2}$ in the $D$-dimensional Minkowski spacetime is given by

$$
f=\frac{\tilde{e}_{1} \tilde{e}_{2}}{r^{D-3}} .
$$

Thus, our normalization of the charges and that of the paper [2] are related as

$$
e^{2}=\frac{\Omega_{(D-2)}}{4 \pi} \cdot \tilde{e}^{2}, \quad \Omega_{2}=4 \pi, \quad \Omega_{3}=2 \pi^{2}, \quad \Omega_{4}=\frac{8 \pi^{2}}{3}, \quad \Omega_{5}=\pi^{3} .
$$


Euclidean space with metric $g_{a b}$ and the dilaton field $\alpha$. In this case the Green function $G\left(x, x^{\prime}\right)$ in the limit $x^{\prime} \rightarrow x$ is nothing but the fluctuations of the field $\psi$. Having in mind this interpretation in what follows we shall use the notation

$$
\left\langle\psi^{2}\right\rangle=\lim _{x, x^{\prime} \rightarrow y} G\left(x, x^{\prime}\right) .
$$

Let us notice that one can also write

$$
\alpha(y)\left\langle\psi^{2}\right\rangle=-\lim _{x, x^{\prime} \rightarrow y} G_{00}\left(x, x^{\prime}\right) .
$$

Thus one can write eq. (2.29) in the form

$$
\mathcal{E}=2 \pi e^{2} \alpha\left\langle\psi^{2}\right\rangle
$$

This representation relates the energy of the point charge $e$ and quantum fluctuations of the $(D-1)$-dimensional Euclidean quantum field $\psi$ with the action eq. (2.17). From $(D-1)$-dimensional point of view $\psi$ is a scalar field.

Of course, the energy of the charge is divergent and it has to be regularized (the subscript $\epsilon$ marks the regularized quantities) either by point splitting or in any other way

$$
\begin{aligned}
\mathcal{E}_{\epsilon} & =2 \pi e^{2} \lim _{x, x^{\prime} \rightarrow y} \sqrt{\alpha(x) \alpha\left(x^{\prime}\right)} G_{\epsilon}\left(x, x^{\prime}\right) \\
& =-2 \pi e^{2} \lim _{x, x^{\prime} \rightarrow y} G_{\epsilon 00}\left(x, x^{\prime}\right) .
\end{aligned}
$$

For our purpose the covariant regularization is preferable. In this case one can put $x^{\prime}=x$ while keeping the ultraviolet (UV) regularization parameter $\epsilon$ fixed and only then take a limit $\epsilon \rightarrow 0$.

\subsection{Self-force}

The self-force acting on a static charge in a static spacetime eq. (2.3) can be defined in terms of an integral of a force density $\mathrm{f}_{\alpha}(X)$ (in a roman font)

$$
\mathrm{f}_{\alpha}=-T_{\alpha \beta} ; \beta
$$

over the constant time slice $\Sigma$. Here $T_{\alpha \beta}$ is the stress-energy tensor of the matter field including the interaction term with the current, but the contribution of the mass distribution is excluded. For a static charge the force has only spatial components $f_{\alpha}=\left(0, f_{a}\right)$. The integral force acting on the static charge distribution can be defined along the lines of the articles $[15,17,20]$ and has the form

$$
f_{a}(y)=\frac{1}{\alpha(y)} \int_{\Sigma} g_{a}{ }^{b}(y, x) \mathrm{f}_{b}(x) \alpha(x) \sqrt{g(x)} d x .
$$

Here $y$ marks the spatial location of the particle and $g_{a}{ }^{b}(y, x)$ is the parallel transport operator within the hypersurface $\Sigma$. It's important to understand that the current density $J^{0}$, the stress-energy tensor $T^{\mu \nu}$, the force density $\mathrm{f}_{\alpha}$, etc. are, in fact, the functions of 
two points in $\Sigma$ : the point $x$ and the position of the particle $y$. For the point charge $J^{0}(x)=J^{0}(x \mid y)=e \delta(x-y) /(\alpha(x) \sqrt{g(x)})$, and the coordinate $y$ enters via the argument of the $\delta$-function.

Substituting $T_{\mu \nu}$ for the electric field eq. (A.29) to eq. (2.34) we get

$$
f_{a}(y)=\frac{1}{\alpha(y)} \int_{\Sigma} g_{a}{ }^{b}(y, x) F_{a 0}(x) J^{0}(x) \alpha(x) \sqrt{g(x)} d x .
$$

Then one can use eq. (2.13) to obtain

$$
f_{a}(y)=\frac{4 \pi}{\alpha(y)} \int_{\mathcal{M}} d x d x^{\prime} \sqrt{g(x)} \sqrt{g\left(x^{\prime}\right)} \alpha(x) \alpha\left(x^{\prime}\right) J^{0}(x) J^{0}\left(x^{\prime}\right) g_{a}{ }^{b}(y, x) \frac{\partial}{\partial x^{b}} G_{00}\left(x, x^{\prime}\right) .
$$

For the point charge the integration of $\delta$-functions over the space leads to a simple result

$$
f_{a}(y)=\left.e^{2} \frac{4 \pi}{\alpha(y)} \frac{\partial}{\partial x^{a}} G_{00}\left(x, x^{\prime}\right)\right|_{x=y, x^{\prime}=y} .
$$

Here the derivative has to be taken before placing points $x$ and $x^{\prime}$ on the worldline of the charge. In coincident points $x=x^{\prime}=y$ the parallel transport operator reduces to the unit matrix $\delta_{a}^{b}$. One can symmetrize the expression in eq. (2.38) to obtain

$$
f_{a}(y)=\left.2 \pi e^{2} \frac{1}{\alpha(y)} \frac{\partial}{\partial x^{a}}\left(G_{00}\left(x, x^{\prime}\right)+G_{00}\left(x^{\prime}, x\right)\right)\right|_{x=y, x^{\prime}=y} .
$$

We rewrite it as

$$
f_{a}(y)=2 \pi e^{2} \frac{1}{\alpha(y)} \frac{\partial}{\partial y^{a}} G_{00}(y, y) .
$$

This expression is formal because it is divergent and has to be properly regularized. Regularization of the self-force can be performed by the same methods as those of the self-energy. Then the regularized self-force acting on the charge can be written in terms of a variation of the self-energy function over the position of the charge.

$$
f_{\epsilon a}=2 \pi e^{2} \frac{1}{\alpha(y)} \partial_{a} G_{\epsilon 00}(y, y)=-2 \pi e^{2} \frac{1}{\alpha(y)} \frac{\partial}{\partial y^{a}}\left(\alpha(y) G_{\epsilon}(y, y)\right)=-\frac{1}{\alpha} \frac{\partial}{\partial y^{a}} \mathcal{E}_{\epsilon} .
$$

Note that $\alpha=1$ at the position of the observer $x_{\mathrm{o}}$ rather than the charge. Therefore, if we want to evaluate the self-force at the position $y$ of the charge, then we can put $\alpha(y)=1$, but only at the very end of computations after taking the derivative over $y$.

\section{Electric charge in the Rindler spacetime}

\subsection{Static Green function}

The $D$-dimensional Rindler metric which describes a static homogeneous gravitational field reads

$$
\begin{aligned}
d s^{2} & =-a^{2} z^{2} d t^{2}+d z^{2}+d \boldsymbol{x}_{\perp}^{2}, \quad d \boldsymbol{x}_{\perp}^{2}=\delta_{i j} d x^{i} d x^{j}, \quad i, j=2, \ldots,(D-1), \\
\alpha & =a z, \quad x \equiv x^{a}=\left(z, x^{i}\right) .
\end{aligned}
$$


We consider a particle at rest at the proper distance $z$ from the horizon. Using the translation invariance of the metric one can always choose $x^{i}=0$. In what follows we assume this choice.

The particle at rest at $z=$ const has a velocity

$$
u^{\alpha}=\frac{1}{a z} \delta_{t}^{\alpha}
$$

and it experiences a constant acceleration

$$
w^{\alpha}=\frac{1}{z} \delta_{z}^{\alpha}
$$

We remind that $t$ is a proper time of an observer at rest at $z=a^{-1}$ and, hence, at this point

$$
w^{\alpha}=a \delta_{z}^{\alpha} .
$$

Our next goal is to find the static electromagnetic Green function in the Rindler spacetime. In a general case one can add to it a solution of the corresponding homogeneous equation. This ambiguity is fixed by a proper choice of the boundary conditions. In our case the homogeneous equation is

$$
(\triangle+V) \psi=\left[\partial_{z}^{2}+\partial_{\boldsymbol{x}_{\perp}}^{2}-\frac{3}{4 z^{2}}\right] \psi=0 .
$$

A solution can be decomposed into modes

$$
\psi \sim \exp \left(i \boldsymbol{k}_{\perp} \boldsymbol{x}_{\perp}\right) Z(z)
$$

where $Z$ is a solution of the equation

$$
\frac{d^{2} Z}{d z^{2}}-\left|\boldsymbol{k}_{\perp}\right|^{2} Z-\frac{3}{4 z^{2}} Z=0 .
$$

This equation has two singular points: horizon $z=0$ and infinity $z=\infty$. Its solution has two arbitrary constants. For $\left|\boldsymbol{k}_{\perp}\right| \neq 0$ one of them is 'killed' by the requirement that the solution is finite at the infinity. Near the horizon one has

$$
Z \sim C_{0} z^{-1 / 2}+C_{1} z^{3 / 2}+
$$

Thus we obtain

$$
A_{0}=-\alpha^{1 / 2} \psi \sim-a^{1 / 2}\left(C_{0}+C_{1} z^{2}\right) \exp \left(i \boldsymbol{k}_{\perp} \boldsymbol{x}_{\perp}\right) .
$$

Keeping the leading term at the horizon one has for $\left|\boldsymbol{k}_{\perp}\right| \neq 0$

$$
F_{\mu \nu} F^{\mu \nu} \sim z^{-2} A_{0, \boldsymbol{x}_{\perp}}^{2} \sim C_{0}^{2} \boldsymbol{k}_{\perp}^{2} z^{-2} .
$$

Thus a regularity of the electromagnetic field at the horizon implies that $C_{0}=0$. To summarize, in a general case a homogeneous solution dependent on $\boldsymbol{x}_{\perp}$ cannot be both regular at the horizon and restricted at infinity. For $\left|\boldsymbol{k}_{\perp}\right|=0$ the regularity of the field strength at the horizon does not restrict constants $C_{0}$ and $C_{1}$. Such a solution is, in fact, a 
zero mode of our field operator. The remaining freedom is a choice of the constant potential value $A_{0}$ at the spatial infinity. But one can always put it equal to zero without changing the strength of the field. Such a solution is unique. This simple analysis shows that if only one finds the static Green function for our problem, which is regular at the horizon and vanishing at the infinity, this solution is unique.

For the Maxwell field we have to find the regularized Green function for the operator eq. (2.9) with the potential eq. (2.11). In the Rindler spacetime $V=-3 /\left(4 z^{2}\right)$ and

$$
(\triangle+V) G\left(x, x^{\prime}\right)=\left[\partial_{z}^{2}+\partial_{\boldsymbol{x}_{\perp}}^{2}-\frac{3}{4 z^{2}}\right] G\left(x, x^{\prime}\right)=-\delta\left(x, x^{\prime}\right) .
$$

We construct the Green function by using the heat kernel $K\left(s \mid x, x^{\prime}\right)$, which is the solution the equation

$$
\left[-\frac{\partial}{\partial s}+\triangle+V\right] K\left(s \mid x, x^{\prime}\right)=0
$$

with the boundary conditions

$$
K\left(0 \mid x, x^{\prime}\right)=\delta\left(x, x^{\prime}\right) .
$$

For the Rindler metric eq. (3.1) $\triangle$ is a flat $(D-1)$-dimensional Laplace operator. The static Green function

$$
G\left(x, x^{\prime}\right)=\int_{0}^{\infty} d s K\left(x, x^{\prime}\right) .
$$

The static Green function in coincidence limit and, hence, the self-energy function diverges. The standard way of extracting the UV divergences is to regularize the heat kernel. In general, the regularized heat kernel can be obtained by multiplying the heat kernel by a weight function $\rho(s, \epsilon)$, which vanishes for $s \ll \epsilon^{2}$ and is equal to one for $s \gg \epsilon^{2}$. For example, a proper time cut-off regularization corresponds to a choice $\rho=\theta\left(s-\epsilon^{2}\right)$. For our calculations it is more convenient to choose

$$
\begin{aligned}
\rho(s, \epsilon) & =\exp \left(-\epsilon^{2} / 4 s\right), \\
K_{\epsilon}\left(s \mid x, x^{\prime}\right) & =\rho(s, \epsilon) K\left(s \mid x, x^{\prime}\right) .
\end{aligned}
$$

The corresponding regularized static Green function reads

$$
G_{\epsilon}\left(x, x^{\prime}\right)=\int_{0}^{\infty} d s K_{\epsilon}\left(s \mid x, x^{\prime}\right) .
$$

Note that this regularization is covariant and can be performed originally in $D$ dimensions for arbitrary spacetime. The result of an integration of this heat kernel over the time $t$ is consistent with the static heat kernel $K_{\epsilon}\left(s \mid x, x^{\prime}\right)$.

One can check that the solution of eqs. (3.12)-(3.13) for the regularized static heat kernel is

$$
K_{\epsilon}\left(s \mid x, x^{\prime}\right)=\sqrt{z z^{\prime}} \frac{2 \pi}{(4 \pi s)^{D / 2}} e^{-\frac{z^{2}+z^{\prime 2}+\boldsymbol{x}_{\perp}^{2}+\epsilon^{2}}{4 s}} I_{1}\left(\frac{z z^{\prime}}{2 s}\right),
$$

where $I_{1}$ is the Bessel function and

$$
\boldsymbol{x}_{\perp}^{2} \equiv \delta_{i j}\left(x^{i}-x^{i}\right)\left(x^{j}-x^{\prime j}\right) .
$$


The choice of the Bessel function $I_{1}$ is dictated by the condition that in the limit of small $s$ the heat kernel eq. (3.18) tends to the regularized heat kernel in the $(D-1)$-dimensional flat space

$$
\left.K_{\epsilon}\right|_{s \rightarrow 0} \rightarrow \frac{1}{(4 \pi s)^{\frac{D}{2}-1}} e^{-\frac{\left(z-z^{\prime}\right)^{2}+\boldsymbol{x}_{\perp}^{2}+\epsilon^{2}}{4 s}} .
$$

The regularized Green function is the integral eq. (3.17) of the regularized heat kernel over the proper time

$$
G_{\epsilon}=\frac{\sqrt{z z^{\prime}}}{2 \pi^{\beta}} I_{1}^{\beta}, \quad \beta=\frac{D}{2}-1,
$$

and

where we defined

$$
G_{\epsilon 00}=-a \frac{z z^{\prime}}{2 \pi^{\beta}} I_{1}^{\beta}
$$

$$
I_{\nu}^{\eta}=\int_{0}^{\infty} u^{\eta-1} e^{-p u} I_{\nu}(c u) d u .
$$

Here are a few useful forms of the integral expressed in terms of the hypergeometric function $F$ or the associated Legendre function $P_{\sigma}^{\rho}$

$$
\begin{aligned}
I_{\nu}^{\eta} & =p^{-\eta-\nu}\left(\frac{c}{2}\right)^{\nu} \frac{\Gamma(\eta+\nu)}{\Gamma(\nu+1)} F\left(\frac{\eta+\nu}{2}, \frac{\eta+\nu+1}{2} ; \nu+1 ; \frac{c^{2}}{p^{2}}\right) \\
& =(p-c)^{-\eta-\nu}\left(\frac{c}{2}\right)^{\nu} \frac{\Gamma(\eta+\nu)}{\Gamma(\nu+1)} F\left(\eta+\nu, \nu+\frac{1}{2} ; 2 \nu+1 ;-\frac{2 c}{p-c}\right) \\
& =e^{-\pi \nu i / 2} \Gamma(\eta+\nu)\left(p^{2}-c^{2}\right)^{-\eta / 2} P_{\eta-1}^{-\nu}\left(\frac{p}{\sqrt{p^{2}-c^{2}}}\right)
\end{aligned}
$$

where

$$
u=\frac{1}{4 s}, \quad p=z^{2}+z^{\prime 2}+x_{\perp}^{2}+\epsilon^{2}, \quad c=2 z z^{\prime} .
$$

Let $R$ and $\bar{R}$ be the distances from the observation point to the charge and its image correspondingly

$$
R^{2}=\left(z-z^{\prime}\right)^{2}+x_{\perp}^{2}, \quad \bar{R}^{2}=\left(z+z^{\prime}\right)^{2}+x_{\perp}^{2} .
$$

The combination $p-c=R^{2}+\epsilon^{2}$ entering the Green function controls its singular behavior in the limit of coincident points. Using the relations

$$
p=\frac{1}{2}\left(\bar{R}^{2}+R^{2}\right)+\epsilon^{2}, \quad c=\frac{1}{2}\left(\bar{R}^{2}-R^{2}\right)
$$

one can express the heat kernel and the Green function in terms of $R$ and $\bar{R}$.

Thus we can write the regularized static Green function in the form

$$
G_{\epsilon 00}=-a \frac{\Gamma(\beta+1)}{2 \pi^{\beta}} \frac{\left(z z^{\prime}\right)^{2}}{\left(R^{2}+\epsilon^{2}\right)^{\beta+1}} F\left(\beta+1, \frac{3}{2} ; 3 ;-\frac{4 z z^{\prime}}{R^{2}+\epsilon^{2}}\right),
$$

One can check that both $G_{\epsilon 00}\left(x, x^{\prime}\right)$ and $G_{\epsilon}\left(x, x^{\prime}\right)$ vanish when one of the arguments, e.g., $x$, is placed on the horizon. It means that they satisfy zero Dirichlet boundary conditions on the Rindler horizon. 
To obtain the general solution for the Green function $G_{00}$ one should remove regularization (put $\epsilon=0$ ) in eq. (3.28) and add a symmetric in $z$ and $z^{\prime}$ zero-mode solution $C_{0}+C_{1}\left(z^{2}+z^{\prime 2}\right)+C_{2} z^{2} z^{\prime 2}$ of a homogeneus equation of the Maxwell equation.

This general solution

$$
G_{00}=-a \frac{\Gamma\left(\frac{D}{2}\right)}{2 \pi^{\frac{D}{2}-1}} \frac{\left(z z^{\prime}\right)^{2}}{R^{D}} F\left(\frac{D}{2}, \frac{3}{2} ; 3 ;-\frac{4 z z^{\prime}}{R^{2}}\right)+C_{0}+C_{1}\left(z^{2}+z^{\prime 2}\right)+C_{2} z^{2} z^{\prime 2}
$$

is parametrized by three arbitrary constants $C_{0}, C_{1}, C_{2}$, which are to be fixed by the boundary conditions at the horizon and at infinity. In our particular case we require $G_{00}$ to be finite, when either $z$ or $z^{\prime}$ are on the horizon, and $G_{00}$ to vanish at infinity. It leads to the choice $C_{0}, C_{1}=C_{2}=0$.

The static Green functions for particular dimensions of the spacetime are given in the appendix B. Their asymptotics at $\epsilon \rightarrow 0$ can be found in appendix C.1.

\subsection{Near-horizon limit of the Schwarzschild black hole}

The geometry of a static black hole near the horizon can be approximated by the Rindler metric. If a charge is close to the horizon its field in the Rindler domain can be described by the above constructed solution. This means that this solution can be obtained as a special limit of the field created by the charge in the black hole geometry. We illustrate this by a simple example of a four dimensional Schwarzschild metric, where the exact solution for the field of a point charge is known. The static Green function near a Schwarzschild black hole of mass $M$ is is [21, 22]

$$
G_{t t}^{\text {Schw }}=-\frac{M}{4 \pi r r^{\prime}}-\frac{(r-M)\left(r^{\prime}-M\right)-M^{2} \cos \theta}{4 \pi r r^{\prime} \sqrt{(r-M)^{2}+\left(r^{\prime}-M\right)^{2}}-2(r-M)\left(r^{\prime}-M\right) \cos \theta-M^{2} \sin ^{2} \theta} .
$$

Here the first term (the Linet term [22]) is regular outside the black hole. It describes the potential of a weakly charged black hole. This term $-M /\left(4 \pi r r^{\prime}\right)$ is important to keep the total charge of the black hole equal to zero and corresponds to the zero mode which is finite on the horizon and vanishes at infinity. In the near horizon limit this term boils down to a trivial constant zero mode in the Rindler space.

Near the horizon the Schwarzschild radial and angle coordinates are related with the proper distance $z$ to the horizon and transverse coordinate $x_{\perp}$ as

$$
\begin{aligned}
r & =2 M\left(1+\frac{z^{2}}{16 M^{2}}-\frac{z^{4}}{768 M^{4}}\right)+O\left(M^{-6}\right), \\
\cos \theta & =1-\frac{\boldsymbol{x}_{\perp}^{2}}{8 M^{2}}+O\left(M^{-4}\right)
\end{aligned}
$$

The Rindler limit can be obtained by taking the limit of large $M$ provided one considers the domain near the horizon, where the proper distances from the horizon to the charge and the observation point are kept fixed and finite. In this limit the coordinate time $x^{0}$ is defined as the red-shifted Schwarzschild time $t$ rescaled in such a way that the timelike Killing vector is normalized to unity when the proper distance to the horizon is equal to $a^{-1}$. 
Then one gets

$$
G_{00}^{\mathrm{Schw}} \approx-\frac{a}{8 \pi} \frac{(R+\bar{R})^{2}}{R \bar{R}},
$$

where $R$ and $\bar{R}$ are given by eq. (3.26).

Our solution eq. (3.29) in four-dimensional Rindler space takes the form

$$
G_{00}=-\frac{a}{8 \pi} \frac{(R-\bar{R})^{2}}{R \bar{R}}
$$

The difference between them is, evidently, the constant zero mode contribution

$$
G_{00}^{\text {Schw }}=G_{00}-\frac{a}{2 \pi} .
$$

Similar calculations can be done for the scalar Green functions. In that case no extra zero mode contributions appear. The difference of the Green functions for the black hole and Rindler spacetimes reflects the difference of their geometry and topology of the horizon.

\section{Electromagnetic self-energy and self-force: results}

\subsection{Divergent part of the static Green function}

In order to derive renormalized self-energy function one has to subtract the UV divergent parts from the Green function eq. (3.21). Their local structure is given by the Hadamard expansion. They can also be deduced from the heat kernel expansion in powers of the proper time $s$. The generic structure of these divergences is

$$
K_{\epsilon}^{\mathrm{div}}\left(s \mid x, x^{\prime}\right)=\frac{e^{-\frac{2 \sigma\left(x, x^{\prime}\right)+\epsilon^{2}}{4 s}-\lambda^{2} s}}{(4 \pi s)^{(D-1) / 2}}\left[a_{0}\left(x, x^{\prime}\right)+s a_{1}\left(x, x^{\prime}\right)+\cdots+s^{[(D-3) / 2]} a_{[(D-3) / 2]}\left(x, x^{\prime}\right)\right] .
$$

Here $a_{n}\left(x, x^{\prime}\right)$ are the Schwinger-DeWitt coefficients. Because we are dealing with the regularized version of the heat kernel, we can safely take first a coincidence limit $x=x^{\prime}$ and then integrate the obtained expression over the proper time $s$

$$
G_{\epsilon}^{\mathrm{div}}(x, x)=\int_{0}^{\infty} d s K_{\epsilon}^{\mathrm{div}}(s \mid x, x),
$$

and finally take an asymptotic of the result at small values of the UV cut-off $\epsilon$. When $x=x^{\prime}$

$$
K_{\epsilon}^{\mathrm{div}}(s \mid x, x)=\frac{e^{-\frac{\epsilon^{2}}{4 s}-\lambda^{2} s}}{(4 \pi s)^{(D-1) / 2}}\left[a_{0}(x, x)+s a_{1}(x, x)+\cdots+s^{[(D-3) / 2]} a_{[(D-3) / 2]}(x, x)\right] .
$$

Here $\lambda$ is an arbitrary IR cut-off parameter. In the case of the electric charge in the Rindler spacetime the first three Schwinger-DeWitt coefficients are

$$
a_{0}(x, x)=1, \quad a_{1}(x, x)=V=-\frac{3}{4 z^{2}}, \quad a_{2}(x, x)=\frac{1}{2} V^{2}+\frac{1}{6} \triangle V=-\frac{15}{32 z^{4}} .
$$

Now expanding $G_{\epsilon}(x, x)$ at small $\epsilon$ and subtracting the $G_{\epsilon}^{\text {div }}(x, x)$ we get

$$
\left\langle\psi^{2}\right\rangle=G_{\epsilon}(y, y)-G_{\epsilon}^{\mathrm{div}}(y, y) .
$$




\subsection{Self-force}

Substitution of the results of the above calculations for different spacetime dimensions (see appendix C.1) into eqs. (2.29) and (2.41) allows one to find both the divergent and the finite residual parts of the self-energy and the self-force. The results can be summarized as follows. One starts with the equation of motion of a neutral particle with mass $m_{0}$

$$
m_{0} a_{\mu}=F_{\mu}^{\mathrm{ext}},
$$

where $F_{\mu}^{\text {ext }}$ is an external force. When the particle has an electric charge $e$ this equation is modified and takes the form

$$
m_{0} a_{\mu}=F_{\mu}^{\mathrm{ext}}+f_{\mu},
$$

where $f_{\mu}$ is an additional 'self-force' proportional to $e^{2}$. The calculations show that it can be presented in the form

$$
f_{\mu}=f_{\mu}^{\mathrm{div}}+f_{\mu}^{\mathrm{res}} .
$$

Here $f_{\mu}^{\text {div }}$ is a divergent in the limit $\epsilon \rightarrow 0$ part of the self-force. It has the following structure

$$
f_{\mu}^{\text {div }}=e^{2} a_{\mu} P(a), \quad P(a)=\sum_{k=0}^{k_{D}} c_{D}^{(2 k)} \frac{a^{2 k}}{\epsilon^{D-3-2 k}} .
$$

Here $D$ is the number of spacetime dimensions and $k_{D}=(D-3) / 2$ for odd $D$ and $k_{D}=$ $D / 2-2$ for even $D$. For odd $D$ in the last term of this expression one should substitute

$$
\frac{1}{\epsilon^{0}} \rightarrow \ln (\epsilon \tilde{\lambda}), \quad \tilde{\lambda}=\lambda e^{\gamma} / 2,
$$

where $\lambda$ is the infrared cut-off and $\gamma$ is the Euler's constant. The numerical coefficients $c_{D}^{(2 k)}$ are given below.

\begin{tabular}{|l|l|l|l|}
\hline$D=4$ & $c_{4}^{(0)}=-\frac{1}{2}$ & & \\
\hline$D=5$ & $c_{5}^{(0)}=-\frac{1}{2 \pi}$ & $c_{5}^{(2)}=\frac{3}{16 \pi}$ & \\
\hline$D=6$ & $c_{6}^{(0)}=-\frac{1}{4 \pi}$ & $c_{6}^{(2)}=-\frac{3}{32 \pi}$ & \\
\hline$D=7$ & $c_{7}^{(0)}=-\frac{1}{2 \pi^{2}}$ & $c_{7}^{(2)}=-\frac{3}{32 \pi^{2}}$ & $c_{7}^{(4)}=\frac{45}{512 \pi^{2}}$ \\
\hline$D=8$ & $c_{8}^{(0)}=-\frac{3}{8 \pi^{2}}$ & $c_{8}^{(2)}=-\frac{3}{64 \pi^{2}}$ & $c_{8}^{(4)}=-\frac{45}{1024 \pi^{2}}$ \\
\hline
\end{tabular}

It should be emphasized that $f_{\mu}^{\text {div }}$ is determined by local terms in the Hadamard expansion of the Green function. We denote by $f_{\mu}^{\text {res }}$ the finite part of the self-force which is the residue obtained by subtracting the local divergent terms from $f_{\mu}$. This part of the force depends on the boundary conditions and, hence, in a general case is non-local. Our calculations give

$$
f_{\mu}^{\text {res }}=e^{2} a_{\mu} a^{D-3}\left[A_{D}+B_{D} \ln (8 \tilde{\lambda} / a)\right] .
$$

For even $D$ the logarithmic terms are absent and one has $B_{D}=0$. The coefficients $A_{D}$ and $B_{D}$ (for odd dimensions) are summarized below. 


\begin{tabular}{|l|l|l|}
\hline$D=4$ & $A_{4}=0$ & $B_{4}=0$ \\
\hline$D=5$ & $A_{5}=\frac{11}{32 \pi}$ & $B_{5}=-\frac{3}{16 \pi}$ \\
\hline$D=6$ & $A_{6}=0$ & $B_{6}=0$ \\
\hline$D=7$ & $A_{7}=\frac{381}{2048 \pi^{2}}$ & $B_{7}=-\frac{45}{512 \pi^{2}}$ \\
\hline$D=8$ & $A_{8}=0$ & $B_{8}=0$ \\
\hline
\end{tabular}

The leading divergence of $P(a)$ is $\sim c_{D}^{(0)} / \epsilon^{D-3}$ and it does not depend on the acceleration. One can absorb it into $m_{0}$, by redefining the mass

$$
m_{0} \rightarrow m=m_{0}-e^{2} \frac{c_{D}^{(0)}}{\epsilon^{D-3}} .
$$

In the four dimensional spacetime this is the only divergent term in the expression for $f_{\mu}^{\text {div }}$, so that one can say that the problem of divergence of the self-force can be solved by a standard method of the mass renormalization, that is by redefining the universal coupling constant in the action for the particle motion. After this one usually says that we must use the action where $m_{0}$ is substituted by $m$, and the value of this parameter $m$ should be determined from observations.

In the higher dimensions one certainly meets a new problem: there exist subleading divergences in the self-force and they depend on the choice of the solution (on the parameter of the acceleration). One may say that these divergences can also be absorbed into a redefinition of the mass of the particle, so that after such a redefinition the equation (4.6) would take the form

$$
m(a) a_{\mu}=F_{\mu}^{\mathrm{ext}}+f_{\mu}^{\mathrm{res}} .
$$

However, in order to obtain such an equation from the least action principle one should modify the action for a free particle. Namely instead of the standard action for a neutral particle

$$
S_{0}=-\frac{m_{0}}{2} \int d \tau \boldsymbol{u}^{2}
$$

one should consider the action

$$
S=S_{0}-\frac{1}{2} \int d \tau Q(a) \boldsymbol{u}^{2}, \quad Q(a)=\sum_{k=0}^{k_{D}} C_{D}^{(2 k)} a^{2 k} .
$$

Here $\boldsymbol{u}$ is the velocity of the particle. For odd $D$ the term $C_{D}^{(D-3)} a^{D-3}$ with highest power of $a$ should be replaced by $\left[C_{D}^{(D-3)}+\tilde{C}_{D}^{(D-3)} \ln (8 \tilde{\lambda} / a)\right] a^{D-3}$. The coefficient $C_{D}^{(0)}$ is nothing but the change of the mass of the particle. The variation of this action results in the following new form of the equation of motion

$$
m_{0} a_{\mu}+\left(Q-a \frac{\partial Q}{\partial a}\right) a_{\mu}+\ldots=F_{\mu}^{\mathrm{ext}}+f_{\mu}^{\mathrm{res}}
$$


The dots denote terms which contain $\dot{a}^{\nu}$ and $\ddot{a}^{\nu}$. These terms vanish for the motion with constant acceleration and the equation takes the form

$$
m_{0} a_{\mu}=\left(a \frac{\partial Q}{\partial a}-Q\right) a_{\mu}+F_{\mu}^{\mathrm{ext}}+f_{\mu}^{\mathrm{res}} .
$$

By comparing this equation with eq. (4.8) and eq. (4.9) one finds that they are the same if

$$
a \frac{\partial Q}{\partial a}-Q=e^{2} P(a)
$$

or, what is equivalent, if one chooses

$$
C_{D}^{(2 k)}=\frac{e^{2}}{2 k-1} \frac{c_{D}^{(2 k)}}{\epsilon^{D-3-2 k}}
$$

Let us make a few comments connected with possible interpretations of the obtained results. One can try to interpret eq. (4.16) as follows. After absorbing the term $Q(a=0)$ into a redefinition (renormalization) of the mass, one may insist that the rest divergent terms in the right-hand side of eq. (4.17) describe a real physical force acting on an accelerated charged particle in the higher dimensions. However, there is another option, which from our point of view is more preferable. Namely, one can assume that in order to have a consistent theory of a particle in the higher dimensions one needs to start with a generalized action of the form eq. (4.15) with arbitrary coefficients $C_{D}^{(2 k)}$. In such a case, in the presence of charge the subleading divergent terms in eq. (4.7) can be absorbed by redefinition of these coefficients by adding the terms of the form eq. (4.19). It should be emphasized that this renormalization procedure does not require the knowledge of the state of motion (acceleration $a$ ). After such a redefinition is performed, following the standard renormalization procedure adopted in the quantum field theory, one may consider $C_{D}^{(2 k)}$ as the finite renormalized coupling constants, the value of which should be found from experiments. This approach was advocated, for example, by Galtsov in $[12,13]$. In such a case the finite non-local contribution to the force $f_{\mu}^{\text {res }}$ can be interpreted as a finite self-force.

\subsection{Self-energy}

After this detailed dicussion of the self-force let us make a few brief comments on the residual (renormalized) value of the self-energy. In four dimensions we reproduce the old result for the self-energy function [6]

$$
\mathcal{E}^{\mathrm{res}}=-\frac{e^{2} a}{2}, \quad D=4
$$

We present here also the results for $E^{\text {res }}$. The residual terms of the self-energy function in other dimensions can be obtained by using appendix C.1.

In even-dimensional spacetimes higher than four dimensions the energy function seems to vanish, though concrete computations we performed for $D=6$ and $D=8$,

$$
\mathcal{E}^{\mathrm{res}}=0, \quad D=6,8 .
$$


In odd-dimensional spacetimes $D=5,7$ we obtain

$$
\begin{aligned}
\mathcal{E}^{\mathrm{res}} & =-\frac{3 e^{2} a}{16 \pi z}\left[\ln (4 \lambda z)+\gamma-\frac{5}{6}\right] & D & =5, \\
\mathcal{E}^{\mathrm{res}} & =-\frac{15 e^{2} a}{512 \pi^{2} z^{3}}\left[\ln (4 \lambda z)+\gamma-\frac{107}{60}\right] & D & =7 .
\end{aligned}
$$

Let us remind that the self-energy functions $\mathcal{E}^{\text {res }}\left(x_{o}, y\right)$ depend on two points, a position of the charge $y^{a}$ and the point of normalization of the Killing vector $x_{o}^{a}$. In the above expressions we use the following choice: $x_{o}^{a}=\left(a^{-1}, x^{i}=0\right)$ and $y^{a}=\left(z, y^{i}=0\right)$. Hence the energy functions are functions of two parameters $a$ and $z$. For the self-energy one has $E^{\text {res }}\left(a^{-1}\right)=\mathcal{E}^{\text {res }}\left(a^{-1}, a^{-1}\right)$. The expression for the self-force of the charge, given by eq. (2.41) calculated at the point $z=a^{-1}$.

\subsection{Summary of results}

The results of the calculations of the residual self-energy $E^{\text {res }}$ and self-force $f_{a}^{\text {res }}$ can be summarized as follows:

- Four dimensions. Self-energy is negative and constant. The self-force vanishes.

$$
E^{\mathrm{res}}=-\frac{e^{2} a}{2}, \quad f_{z}^{\mathrm{res}}=0 .
$$

- Five dimensions.

$$
\begin{aligned}
E^{\mathrm{res}} & =-\frac{3 e^{2} a^{2}}{16 \pi}\left[\ln \left(\frac{4 \lambda}{a}\right)+\gamma-\frac{5}{6}\right], \\
f_{z}^{\mathrm{res}} & =-\frac{3 e^{2} a^{3}}{16 \pi}\left[\ln \left(\frac{4 \lambda}{a}\right)+\gamma-\frac{11}{6}\right] .
\end{aligned}
$$

- Six dimensions

$$
E^{\mathrm{res}}=0, \quad f_{z}^{\mathrm{res}}=0
$$

- Seven dimensions.

$$
\begin{aligned}
E^{\mathrm{res}} & =-\frac{15 e^{2} a^{4}}{512 \pi^{2}}\left[\ln \left(\frac{4 \lambda}{a}\right)+\gamma-\frac{107}{60}\right] \\
f_{z}^{\mathrm{res}} & =-\frac{45 e^{2} a^{5}}{512 \pi^{2}}\left[\ln \left(\frac{4 \lambda}{a}\right)+\gamma-\frac{127}{60}\right] .
\end{aligned}
$$

- Eight dimensions

$$
E^{\mathrm{res}}=0, \quad f_{z}^{\mathrm{res}}=0 .
$$

The invariant self-force is equal to the absolute value of the $z$-component of the self-force $f^{\text {res }}=\sqrt{f_{a}^{\text {res }} f^{\text {res } a}}=\left|f_{z}^{\text {res }}\right|$.

One can see that the residual self-force in even dimensions vanishes. We obtained this result for $D \leq 8$, however one can make a conjecture that this result is valid in any even 
dimensional spacetimes with a static homogeneous gravitational field. Let us also notice that in odd dimensions the residual self-energy and the residual self-force depend on the IR cutoff $\lambda=1 / l$ which has the dimensionality of inverse length. A similar logarithmic terms are also present in the expression for the self-force of a charge near a five-dimensional static black hole (see discussion in [2]).

\section{A scalar charge in a static spacetime}

\subsection{Equations}

Let us consider now a self-energy of a scalar charge in a static spacetimes. A minimally coupled massless scalar field $\Phi$ is described by an action

$$
I=-\frac{1}{8 \pi} \int d X \sqrt{-g^{\mathrm{D}}} \Phi^{; \mu} \Phi_{; \mu}+\int d X \sqrt{-g^{\mathrm{D}}} J \Phi .
$$

It obeys the equation

$$
\square \Phi=-4 \pi J
$$

The energy function $\mathcal{E}$ of a static configuration of the scalar field is given by the integral eq. (2.14) of the stress-energy tensor of the scalar field eq. (A.39). Taking into account only the scalar field contribution, we have

$$
T_{0}^{0}=\frac{1}{8 \pi} \Phi^{; a} \Phi_{; a}+\Phi J .
$$

The expression for the energy can be written as

$$
\mathcal{E}=-\frac{1}{2} \int d x \sqrt{g} \alpha J \Phi-\frac{1}{8 \pi} \int d x \partial_{a}\left(\sqrt{g} \alpha \Phi \Phi^{; a}\right)
$$

The last term is proportional to the surface integral of $\Phi \Phi^{; a}$ over the boundary. It vanishes because of the boundary conditions at infinity and on the horizon.

Similarly to the electromagnetic case it is useful to introduce another field variable $\varphi$

$$
\Phi=\alpha^{-1 / 2} \varphi
$$

The field $\varphi$ satisfies the equation

$$
\begin{aligned}
(\triangle+V) \varphi & =-4 \pi j, \\
V & =\frac{(\nabla \alpha)^{2}}{4 \alpha^{2}}-\frac{\triangle \alpha}{2 \alpha} \equiv-\frac{\triangle\left(\alpha^{1 / 2}\right)}{\alpha^{1 / 2}}, \\
j & =\alpha^{1 / 2} J .
\end{aligned}
$$

Here

$$
\triangle=g^{a b} \nabla_{a} \nabla_{b} .
$$




\section{$5.2 \quad$ Self-energy}

In terms of this field the energy can be rewritten in the form

$$
\mathcal{E}=-\frac{1}{8 \pi} \int d x \sqrt{g} g^{a b}\left(\varphi_{, a}-\frac{\alpha_{, a}}{2 \alpha} \varphi\right)\left(\varphi_{, b}-\frac{\alpha_{, b}}{2 \alpha} \varphi\right) .
$$

This expression for the energy can be interpreted as the Euclidean action of a $(D-1)$ dimensional scalar field $\varphi$ interacting with the external dilaton field $\alpha$. One can use this analogy to reformulate the problem of calculation of the self-energy of a point charge in terms of the Euclidean quantum field theory defined on $(D-1)$-dimensional space and in the presence of the external dilaton field.

For a point scalar charge $q$ located at $y$ the charge distribution reads

$$
J=q \frac{1}{\sqrt{g}} \delta(x-y) .
$$

Thus, the self-energy function of point scalar charges can be written in the form [8]

$$
\mathcal{E}=-2 \pi q^{2} \alpha(y) G(y, y)=-2 \pi q^{2} \alpha(y)\left\langle\varphi^{2}\right\rangle .
$$

Here $G(y, y)$ is the coincidence limit $x, x^{\prime} \rightarrow y$ of the regularized Green function. The Green function $G$ corresponds to the operator eq. (5.6)

$$
(\triangle+V) G\left(x, x^{\prime}\right)=-\delta\left(x, x^{\prime}\right)
$$

For point sources energy function $\mathcal{E}$ diverges. To deal with this divergence one has to use some regularization and renormalization schemes. Following the same arguments as in electromagnetic case we obtain

$$
\mathcal{E}_{\epsilon}=-2 \pi q^{2} \lim _{x, x^{\prime} \rightarrow y} \sqrt{\alpha(x) \alpha\left(x^{\prime}\right)} G_{\epsilon}\left(x, x^{\prime}\right) .
$$

\subsection{Self-force}

The self-force of the static scalar charge distribution $J$ in the static spacetime can be defined $[15,23]$ exactly in the same way as that of the electric charge distribution eqs. (2.35)(2.34).

$$
\begin{aligned}
f_{a}(y) & =\frac{1}{\alpha(y)} \int_{\Sigma} g_{a}{ }^{b}(y, x) \mathrm{f}_{b}(x) \alpha(x) \sqrt{g(x)} d x \\
\mathrm{f}_{\alpha} & =-T_{\alpha \beta} ; \beta
\end{aligned}
$$

The stress-energy tensor of the scalar charge eq. (A.39) without mass terms reads

$$
T^{\mu \nu}=\frac{1}{4 \pi}\left(\Phi^{; \mu} \Phi^{; \nu}-\frac{1}{2} g^{\mathrm{D} \mu \nu} \Phi^{; \alpha} \Phi_{; \alpha}\right)-u^{\mu} u^{\nu} \Phi J .
$$

The divergence of the stress-energy tensor gives the density of the self-force

$$
\mathrm{f}_{\alpha}=\Phi J w_{\alpha}+J\left(\delta_{\alpha}^{\beta}+u_{\alpha} u^{\beta}\right) \nabla_{\beta} \Phi,
$$


where

$$
w_{\alpha}=u^{\sigma} \nabla_{\sigma} u_{\alpha}
$$

is the acceleration vector corresponding to the worldline with the velocity $u^{\alpha}$.

We define the self-force density of the scalar charge distribution as the right-hand side of the equation of motion eq. (A.37)

$$
\mu w_{\alpha}=\mathrm{f}_{\alpha} .
$$

Here $\mu$ is the mass density of the source and

$$
\mathrm{f}_{\alpha}=J\left(\delta_{\alpha}^{\beta}+u_{\alpha} u^{\beta}\right) \nabla_{\beta} \Phi+w_{\alpha} \Phi J .
$$

This force, evidently, is orthogonal to the velocity $u^{\alpha}$ of the charge. So, for the $T^{\mu \nu}$ in the form eq. (5.17) the problem of dependence of the mass of the point scalar charge on its proper time, discussed in [23], does not appear.

Note that the force density eq. (5.21) is the sum of terms of two kinds. The first one is determined by a gradient of the scalar field, while the second term in $f_{\alpha}$ is proportional to the acceleration vector and to the value of the scalar field. The second term has the structure similar to the contribution of the mass density, though it depends on the value of the scalar field at the position of the charge. This similarity is sometimes used to move this term to the left-hand side of the motion equation and redefine the inertial mass density of the charge and the force (see, e.g., [17] and references therein) as

$$
\begin{aligned}
\tilde{\mu} & =\mu-J \Phi, \\
\tilde{\mathrm{f}}_{\alpha} & =\mathrm{f}_{\alpha}-w_{\alpha} \Phi J .
\end{aligned}
$$

In this representation the force $\tilde{\mathrm{f}}_{\alpha}$ depends only on $\nabla_{\beta} \Phi[2,17,19,23]$

$$
\tilde{\mathrm{f}}_{\alpha}=J\left(\delta_{\alpha}^{\beta}+u_{\alpha} u^{\beta}\right) \nabla_{\beta} \Phi,
$$

but in the general case the 'inertial' mass $\tilde{\mu}$ is position dependent.

In this paper we use the motion equations eqs. (5.20), (5.21), (A.37) in their original form, that is without any redefinition of the mass and the force. This choice guarantees an agreement between the change of the self-energy function of the charge and the work of the self-force during adiabatic displacement of the charge. At the end of this section we provide the results for both kinds of forces.

In the case of a static charge in the static spacetime eq. (2.3) the expression for self-force reads

$$
\begin{aligned}
f_{a}(y) & =-\frac{1}{\alpha(y)} \int_{\Sigma} g_{a}{ }^{b}(y, x) T_{b \beta}{ }^{; \beta}(x) \alpha(x) \sqrt{g(x)} d x \\
& =\frac{1}{\alpha(y)} \int_{\Sigma} g_{a}{ }^{b}(y, x)\left(J \nabla_{b} \Phi+J u_{b} u^{\sigma} \nabla_{\sigma} \Phi+w_{b} J \Phi\right) \alpha(x) \sqrt{g(x)} d x .
\end{aligned}
$$

Its velocity is proportional to the Killing vector, while the acceleration is $w_{\mu}=\alpha^{-1} \partial_{\mu} \alpha$. The time component of the self-force and $\nabla_{0} \Phi$ vanish, and we obtain

$$
f_{a}(y)=\frac{1}{\alpha(y)} \int_{\Sigma} d x \sqrt{g} \alpha g_{a}{ }^{b}(y, x)\left(J \nabla_{b} \Phi+w_{b} J \Phi\right) .
$$


Substituting eq. (5.5) and eq. (5.8) in this relation one gets

$$
\begin{aligned}
f_{a}(y) & =\frac{1}{\alpha(y)} \int_{\Sigma} d x \sqrt{g} g_{a}{ }^{b}(y, x)\left(j \nabla_{b} \varphi+\frac{1}{2} w_{b} j \varphi\right) \\
& =\frac{4 \pi}{\alpha(y)} \int_{\Sigma} d x d x^{\prime} \sqrt{g(x)} \sqrt{g\left(x^{\prime}\right)} j(x) j\left(x^{\prime}\right) g_{a}{ }^{b}(y, x)\left(\frac{\partial}{\partial x^{b}} G\left(x, x^{\prime}\right)+\frac{1}{2} w_{b} G\left(x, x^{\prime}\right)\right) \\
& =\frac{4 \pi}{\alpha(y)} \int_{\Sigma} d x d x^{\prime} \sqrt{g(x)} \sqrt{g\left(x^{\prime}\right)} \frac{j(x) j\left(x^{\prime}\right)}{\sqrt{\alpha(x)} \sqrt{\alpha\left(x^{\prime}\right)}} g_{a}{ }^{b}(y, x) \frac{\partial}{\partial x^{b}}\left(\sqrt{\alpha(x)} \sqrt{\alpha\left(x^{\prime}\right)} G\left(x, x^{\prime}\right)\right) .
\end{aligned}
$$

For a point charge located at $y$ the charge density distribution is

$$
j=q \frac{\sqrt{\alpha}}{\sqrt{g}} \delta(x-y) .
$$

So that one finally obtains

$$
f_{a}(y)=\left.\frac{4 \pi q^{2}}{\alpha(y)} \frac{\partial}{\partial x^{a}}\left(\sqrt{\alpha(x)} \sqrt{\alpha\left(x^{\prime}\right)} G\left(x, x^{\prime}\right)\right)\right|_{x=y, x^{\prime}=y} .
$$

The derivative has to be taken before placing points $x$ and $x^{\prime}$ on the worldline of the charge. This divergent expression has to be regularized. Following the lines of the electromagnetic case one can rewrite it formally as

$$
f_{\epsilon a}(y)=2 \pi q^{2} \frac{1}{\alpha(y)} \frac{\partial}{\partial y^{a}}\left(\alpha(y) G_{\epsilon}(y, y)\right) .
$$

Comparing this formula with the expression for the self-energy function eq. (5.12) one can see that

$$
f_{a}=-\frac{1}{\alpha} \partial_{a} \mathcal{E}
$$

Thus, the relation between the self-force and the variation of the self-energy function over the position of the charge is the same as in the electromagnetic case. This fact is not surprising but it's a good test of the validity of derivation of the self-force. ${ }^{4}$

\section{Scalar charge in the Rindler spacetime}

For the scalar field in the $D$-dimensional Rindler space we have to find the regularized Green function and the heat kernel for the operator $\triangle+V$, where $\triangle$ is the flat $(D-1)$ dimensional flat Laplace operator and the potential $V=1 /\left(4 z^{2}\right)$

$$
(\triangle+V) G\left(x, x^{\prime}\right)=\left[\partial_{z}^{2}+\partial_{\boldsymbol{x}_{\perp}}^{2}+\frac{1}{4 z^{2}}\right] G\left(x, x^{\prime}\right)=-\delta\left(x, x^{\prime}\right)
$$

\footnotetext{
${ }^{4}$ In the articles $[2,17,19,23]$ the modified self-force $\tilde{f}_{\mu}$ has been used. It can be derived by integrating densities eqs. (5.23) and (5.24). The result of computations can be presented in the form

$$
\tilde{f}_{\mu}=f_{\mu}+\frac{2}{\alpha} w_{\mu} \mathcal{E}=-\frac{1}{\alpha}\left(\partial_{\mu} \mathcal{E}-2 w_{\mu} \mathcal{E}\right)
$$

This formula is applicable to static scalar charges in arbitrary static spacetimes.
} 
Choosing the same regularization as for Maxwell field we get

$$
K_{\epsilon}\left(s \mid x, x^{\prime}\right)=\rho(s, \epsilon) K\left(s \mid x, x^{\prime}\right), \quad \rho(s, \epsilon)=\exp \left(-\epsilon^{2} / 4 s\right) .
$$

The regularized static Green function is

$$
G_{\epsilon}\left(x, x^{\prime}\right)=\int_{0}^{\infty} d s K_{\epsilon}\left(s \mid x, x^{\prime}\right) .
$$

One can check that the solution for the regularized static heat kernel is

$$
K_{\epsilon}=\sqrt{z z^{\prime}} \frac{2 \pi}{(4 \pi s)^{D / 2}} e^{-\frac{z^{2}+z^{\prime 2}+\boldsymbol{x}_{\perp}^{2}+\epsilon^{2}}{4 s}} I_{0}\left(\frac{z z^{\prime}}{2 s}\right)
$$

where $I_{0}$ is the Bessel function and $\boldsymbol{x}_{\perp}^{2} \equiv g_{i j}\left(x^{i}-x^{\prime i}\right)\left(x^{j}-x^{\prime j}\right)$.

The regularized Green function is the integral eq. (6.3) of the regularized heat kernel over the proper time

$$
G_{\epsilon}=\frac{\sqrt{z z^{\prime}}}{2 \pi^{\beta}} I_{0}^{\beta}, \quad \beta=\frac{D}{2}-1,
$$

where $I_{\nu}^{\eta}$ is defined in eq. (3.24). The regularized static scalar Green function can be written in the exact closed form

$$
G_{\epsilon}=\frac{\Gamma(\beta)}{2 \pi^{\beta}} \frac{\left(z z^{\prime}\right)^{1 / 2}}{\left(R^{2}+\epsilon^{2}\right)^{\beta}} F\left(\beta, \frac{1}{2} ; 1 ;-\frac{4 z z^{\prime}}{R^{2}+\epsilon^{2}}\right),
$$

where $R$ is given by eq. (3.26).

Note that the static Green function for the original scalar field $\Phi$ (see eq. (5.2)), differs from $G_{\epsilon}$ by the factor $\left(z z^{\prime}\right)^{-1 / 2}$

$$
\mathbb{G}\left(x, x^{\prime}\right)=\left\langle\Phi(x) \Phi\left(x^{\prime}\right)\right\rangle=\left(z z^{\prime}\right)^{-1 / 2} G\left(x, x^{\prime}\right) .
$$

Along the lines of the electromagnetic case, one can obtain the general solution for the Green function $\mathbb{G}\left(x, x^{\prime}\right)$ from eq. (6.7) if one adds zero-mode solutions parametrized by three arbitrary constants $C_{0}+C_{1} \ln \left(z z^{\prime}\right)+C_{2} \ln z \ln z^{\prime}$. This general solution reads

$$
\mathbb{G}\left(x, x^{\prime}\right)=\frac{\Gamma\left(\frac{D}{2}-1\right)}{2 \pi^{\frac{D}{2}-1}} \frac{1}{R^{D-2}} F\left(\frac{D}{2}-1, \frac{1}{2} ; 1 ;-\frac{4 z z^{\prime}}{R^{2}}\right)+C_{0}+C_{1} \ln \left(z z^{\prime}\right)+C_{2} \ln z \ln z^{\prime} .
$$

The three arbitrary constants $C_{0}, C_{1}, C_{2}$ are to be fixed by the boundary conditions when $x$ or $x^{\prime}$ are at the horizon and at infinity. Analysis, similar to that of the solution for the vector potential of the electric charge, shows that regular at the horizon and vanishing at infinity scalar field configuration corresponds to $C_{0}=C_{1}=C_{2}=0$. One can check that $G_{\epsilon}\left(x, x^{\prime}\right)$ given by eq. (6.6) vanishes when one of the arguments, e.g., $x$, is placed on the horizon. It means that it satisfies zero Dirichlet boundary conditions on the Rindler horizon. The static Green functions for particular dimensions of the spacetime are given in the appendix B. Their asymptotics at $\epsilon \rightarrow 0$ are in appendix C.2. 


\section{Scalar self-energy and self-force: results}

\subsection{Divergent part of the static Green function}

To renormalize the self-energy one has to subtract the UV divergent parts from the Green function eq. (6.5). The local structure of divergences is given by the first terms in expansion eq. (4.3) of the heat kernel over the proper time parameter. In the case of the scalar charge in the Rindler spacetime the corresponding Schwinger-DeWitt coefficients are

$$
a_{0}(x, x)=1, \quad a_{1}(x, x)=V=\frac{1}{4 z^{2}}, \quad a_{2}(x, x)=\frac{1}{2} V^{2}+\frac{1}{6} \triangle V=\frac{9}{32 z^{4}} .
$$

The residual finite value for quantum fluctuations of the field $\varphi$ at the point $y$ reads

$$
\left\langle\varphi^{2}\right\rangle^{\mathrm{res}}=G_{\epsilon}(y, y)-G_{\epsilon}{ }^{\mathrm{div}}(y, y),
$$

which enters the formula eq. (5.12) for the self-energy of the charged particle. For different spacetime dimensions (see appendix C.2) we finally obtain the self-energy function of the scalar charge which has the constant acceleration.

\subsection{Self-force}

The divergent and finite parts of the residual self-force acting on the scalar charge can be computed along the same lines as in the electromagnetic case (section 4.2)

$$
m_{0} a_{\mu}=F_{\mu}^{\mathrm{ext}}+f_{\mu}, \quad f_{\mu}=f_{\mu}^{\mathrm{div}}+f_{\mu}^{\mathrm{res}} .
$$

Here $f_{\mu}^{\text {div }}$ is a divergent in the limit $\epsilon \rightarrow 0$ part of the self-force and $f_{\mu}^{\text {res }}$ is the finite residual part of the self-force. They can be derived from the corresponding divergent and residual finite parts of the self-energy using the relations (see also eq. (2.41))

$$
f_{\epsilon a}=-\frac{1}{\alpha} \frac{\partial}{\partial y^{a}} \mathcal{E}_{\epsilon}, \quad f_{a}^{\mathrm{div}}=-\frac{1}{\alpha} \frac{\partial}{\partial y^{a}} \mathcal{E}^{\mathrm{div}}, \quad f_{a}^{\mathrm{res}}=-\frac{1}{\alpha} \frac{\partial}{\partial y^{a}} \mathcal{E}^{\mathrm{res}} .
$$

Note that in the Rindler spacetime $\alpha=a z$. It is equal to one at the point of observation.

It should be emphasized that in this approach the bare mass $m_{0}$, or its renormalized version $m$ (see eq. (4.12)), are constants. In the literature on the self-force of scalar charges $[2,12,13,17,19,23]$ the same motion equation eq. (7.3) is often presented in the form where some specific, proportional to the acceleration, part of $f_{a}$ is moved to the left-hand side of the motion equation and included into the redefinition of the inertial mass (see eqs. (5.22)-(5.24)), which then becomes dependent on the position of the scalar charge. We denote the corresponding remaining part of the self-force in the motion equation as $\tilde{f}_{a}$. It is related to the self-energy function as

$$
\tilde{f}_{a}^{\mathrm{div}}=-\frac{1}{\alpha}\left(\frac{\partial}{\partial y^{a}} \mathcal{E}^{\mathrm{div}}-2 w_{a} \mathcal{E}^{\mathrm{div}}\right), \quad \tilde{f}_{a}^{\mathrm{res}}=-\frac{1}{\alpha}\left(\frac{\partial}{\partial y^{a}} \mathcal{E}^{\mathrm{res}}-2 w_{a} \mathcal{E}^{\mathrm{res}}\right) .
$$

The divergent part of the self-force has the following structure

$$
f_{\mu}^{\text {div }}=q^{2} a_{\mu} P(a), \quad P(a)=\sum_{k=0}^{k_{D}} c_{D}^{(2 k)} \frac{a^{2 k}}{\epsilon^{D-3-2 k}} .
$$


Here $k_{D}=(D-3) / 2$ for odd $D$ and $k_{D}=D / 2-2$ for even $D$. For odd $D$ in the last term of this expression one should make a substitution

$$
\frac{1}{\epsilon^{0}} \rightarrow \ln (\epsilon \tilde{\lambda}), \quad \tilde{\lambda}=\lambda e^{\gamma} / 2,
$$

where $\lambda$ is the infrared cut-off and $\gamma$ is the Euler's constant. The numerical coefficients $c_{D}^{(2 k)}$ are

\begin{tabular}{|l|l|l|l|}
\hline$D=4$ & $c_{4}^{(0)}=\frac{1}{2}$ & & \\
\hline$D=5$ & $c_{5}^{(0)}=\frac{1}{2 \pi}$ & $c_{5}^{(2)}=\frac{1}{16 \pi}$ & \\
\hline$D=6$ & $c_{6}^{(0)}=\frac{1}{4 \pi}$ & $c_{6}^{(2)}=-\frac{1}{32 \pi}$ & \\
\hline$D=7$ & $c_{7}^{(0)}=\frac{1}{2 \pi^{2}}$ & $c_{7}^{(2)}=-\frac{1}{32 \pi^{2}}$ & $c_{7}^{(4)}=\frac{27}{512 \pi^{2}}$ \\
\hline$D=8$ & $c_{8}^{(0)}=\frac{3}{8 \pi^{2}}$ & $c_{8}^{(2)}=-\frac{1}{64 \pi^{2}}$ & $c_{8}^{(4)}=-\frac{27}{1024 \pi^{2}}$ \\
\hline
\end{tabular}

The finite residual part of the self-force has the structure

$$
f_{\mu}^{\mathrm{res}}=q^{2} a_{\mu} a^{D-3}\left[A_{D}+B_{D} \ln (8 \tilde{\lambda} / a)\right] .
$$

For even $D$ the logarithmic terms are absent and one has $B_{D}=0$. Our calculations lead to the following the coefficients $A_{D}$ and $B_{D}$

\begin{tabular}{|l|l|l|}
\hline$D=4$ & $A_{4}=0$ & $B_{4}=0$ \\
\hline$D=5$ & $A_{5}=\frac{5}{32 \pi}$ & $B_{5}=-\frac{1}{16 \pi}$ \\
\hline$D=6$ & $A_{6}=0$ & $B_{6}=0$ \\
\hline$D=7$ & $A_{7}=\frac{243}{2048 \pi^{2}}$ & $B_{7}=-\frac{27}{512 \pi^{2}}$ \\
\hline$D=8$ & $A_{8}=0$ & $B_{8}=0$ \\
\hline
\end{tabular}

Similarly we obtain the coefficients for the divergent and finite residual parts of $\tilde{f}_{a}$

$$
\tilde{f}_{\mu}^{\mathrm{div}}=q^{2} a_{\mu} \tilde{P}(a), \quad \tilde{P}(a)=\sum_{k=0}^{k_{D}} \tilde{c}_{D}^{(2 k)} \frac{a^{2 k}}{\epsilon^{D-3-2 k}}
$$

\begin{tabular}{|l|l|l|l|}
\hline$D=4$ & $\tilde{c}_{4}^{(0)}=-\frac{1}{2}$ & & \\
\hline$D=5$ & $\tilde{c}_{5}^{(0)}=-\frac{1}{2 \pi}$ & $\tilde{c}_{5}^{(2)}=\frac{3}{16 \pi}$ & \\
\hline$D=6$ & $\tilde{c}_{6}^{(0)}=-\frac{1}{4 \pi}$ & $\tilde{c}_{6}^{(2)}=-\frac{3}{32 \pi}$ & \\
\hline$D=7$ & $\tilde{c}_{7}^{(0)}=-\frac{1}{2 \pi^{2}}$ & $\tilde{c}_{7}^{(2)}=-\frac{3}{32 \pi^{2}}$ & $\tilde{c}_{7}^{(4)}=\frac{45}{512 \pi^{2}}$ \\
\hline$D=8$ & $\tilde{c}_{8}^{(0)}=-\frac{3}{8 \pi^{2}}$ & $\tilde{c}_{8}^{(2)}=-\frac{3}{64 \pi^{2}}$ & $\tilde{c}_{8}^{(4)}=-\frac{45}{1024 \pi^{2}}$ \\
\hline
\end{tabular}


The finite residual part of the tilde-self-force has the structure

$$
\tilde{f}_{\mu}^{\text {res }}=q^{2} a_{\mu} a^{D-3}\left[\tilde{A}_{D}+\tilde{B}_{D} \ln (8 \tilde{\lambda} / a)\right] .
$$

\begin{tabular}{|l|l|l|}
\hline$D=4$ & $\tilde{A}_{4}=0$ & $\tilde{B}_{4}=0$ \\
\hline$D=5$ & $\tilde{A}_{5}=\frac{11}{32 \pi}$ & $\tilde{B}_{5}=-\frac{3}{16 \pi}$ \\
\hline$D=6$ & $\tilde{A}_{6}=0$ & $\tilde{B}_{6}=0$ \\
\hline$D=7$ & $\tilde{A}_{7}=\frac{381}{2048 \pi^{2}}$ & $\tilde{B}_{7}=-\frac{45}{512 \pi^{2}}$ \\
\hline$D=8$ & $\tilde{A}_{8}=0$ & $\tilde{B}_{8}=0$ \\
\hline
\end{tabular}

One can see an amazing coincidence of electromagnetic residual self-forces $f_{\mu}^{\text {div }}$ and $f_{\mu}^{\text {res }}$ (see eqs. (4.9) and (4.11)) with the scalar ones $\tilde{f}_{\mu}^{\text {div }}$ and $\tilde{f}_{\mu}^{\text {res }}$ (see eqs. (7.9) and (7.10)).

\subsection{Self-energy}

In even dimensions $D=4,6,8$, etc. self-energy function vanishes. In odd-dimensional spacetimes we have

$$
\begin{array}{ll}
\mathcal{E}=-\frac{q^{2} a}{16 \pi z}\left[\ln (4 \lambda z)+\gamma-\frac{3}{2}\right] & D=5, \\
\mathcal{E}=-\frac{9 q^{2} a}{512 \pi^{2} z^{3}}\left[\ln (4 \lambda z)+\gamma-\frac{23}{12}\right] & D=7 .
\end{array}
$$

As earlier the self-energy functions $\mathcal{E}^{\text {res }}\left(x_{o}, y\right)$ depend on two points, a position of the charge $y^{a}$ and the point of normalization of the Killing vector $x_{o}^{a}$. We put $x_{o}^{a}=\left(a^{-1}, x^{i}=0\right)$ and $y^{a}=\left(z, y^{i}=0\right)$ and wrote the residual self-energy functions as functions two parameters $a$ and $z$. For the residual self-energy one has $E^{\text {res }}\left(a^{-1}\right)=\mathcal{E}^{\text {res }}\left(a^{-1}, a^{-1}\right)$. The residual self-force of the charge is calculated at the point $z=a^{-1}$.

\subsection{Summary of results}

The results of the calculations of the finite residual self-energy $E^{\text {res }}$ and self-forces $f_{a}^{\text {res }}$ and $\tilde{f}_{a}^{\text {res }}$ can be summarized as follows:

- Four dimensions. The self-energy and self-forces vanish.

$$
E^{\text {res }}=0, \quad f_{z}^{\text {res }}=0, \quad \tilde{f}_{z}^{\text {res }}=0 .
$$

- Five dimensions.

$$
\begin{aligned}
E^{\mathrm{res}} & =-\frac{q^{2} a^{2}}{16 \pi}\left[\ln \left(\frac{4 \lambda}{a}\right)+\gamma-\frac{3}{2}\right], \\
f_{z}^{\mathrm{res}} & =-\frac{q^{2} a^{3}}{16 \pi}\left[\ln \left(\frac{4 \lambda}{a}\right)+\gamma-\frac{5}{2}\right], \\
\tilde{f}_{z}^{\mathrm{res}} & =-\frac{3 q^{2} a^{3}}{16 \pi}\left[\ln \left(\frac{4 \lambda}{a}\right)+\gamma-\frac{11}{6}\right] .
\end{aligned}
$$


- Six dimensions. The self-energy and self-force vanish

$$
E^{\text {res }}=0, \quad f_{z}^{\text {res }}=0, \quad \tilde{f}_{z}^{\text {res }}=0 .
$$

- Seven dimensions.

$$
\begin{aligned}
E^{\mathrm{res}} & =-\frac{9 q^{2} a^{4}}{512 \pi^{2}}\left[\ln (4 \lambda z)+\gamma-\frac{23}{12}\right], \\
f_{z}^{\mathrm{res}} & =-\frac{27 q^{2} a^{5}}{512 \pi^{2}}\left[\ln \left(\frac{4 \lambda}{a}\right)+\gamma-\frac{9}{4}\right], \\
\tilde{f}_{z}^{\text {res }} & =-\frac{45 q^{2} a^{5}}{512 \pi^{2}}\left[\ln \left(\frac{4 \lambda}{a}\right)+\gamma-\frac{127}{60}\right] .
\end{aligned}
$$

- Eight dimensions

$$
E^{\mathrm{res}}=0, \quad f_{z}^{\mathrm{res}}=0, \quad \tilde{f}_{z}^{\mathrm{res}}=0 .
$$

Similar to the electric charge the self-force for the scalar charge vanishes in even dimensions. In odd dimensions the self-energy and the self-force depend on the IR cutoff $\lambda$ that needs to be chosen from physical arguments (see discussion of this problem, e.g., in $[2])$.

\section{Discussion}

In the present paper we calculated a self-energy and a self-force for a point charge in a higher-dimensional static homogeneous gravitational field.

One of the results, presented in the present paper, seems to be quite interesting and intriguing. Namely, we demonstrated that in even dimensions $4 \leq D \leq 8$ the finite residual part of the self-force for both electric and scalar charge identically vanishes. We do not know a deep reason for this, but one might make the conjecture, that is a common feature of even dimensional spacetimes with the number of dimensions greater than or equal to four.

In 4 dimensions this result can be expected for the following reason. The invariant residual self-force acting on a charge $e$ near a Schwarzschild or a Reissner-Nordström black hole of mass $M$ is $[4,5,17]$

$$
f^{\mathrm{res}}=\left(f^{\mathrm{sel} f \mu} f_{\mu}^{\mathrm{res}}\right)^{1 / 2}=\frac{e^{2} M}{r^{3}} .
$$

In order to pass to the Rindler limit in this formula one needs to take the limit, when the gravitational radius $r_{g}=2 M$ infinitely grows while the proper distance to the horizon is fixed. In this limit $f^{\text {res }}=0$ that coincides with our result. In the higher even dimensions the vanishing of the residual self-force in the Rindler spacetime implies that the corresponding invariant of this self-force for the charge near the black hole remains finite on the horizon. This result is valid both for electric and scalar charges.

The results obtained for a static homogeneous gravitational field can be directly applied without any changes to the case of a uniformly accelerated motion of the charge in the 
higher dimensional Minkowski spacetime. In four dimensions the vanishing of the resudual self-force acting on a uniformly accelerated charge is well known property (see e.g. [24, 25]). The obtained in this paper results indicate that a similar property is valid for accelerated scalar and electric charges in any even dimensional Minkowski spacetime.

A natural question arises about the validity of the calculations and the results. For example, one can ask the question: at what distance from the horizon the residual self-force of the charge becomes comparable with the gravitational attraction of the particle with the mass $m$. Let us neglect for a moment logarithmic factors. The finite part of the self-force is of the order of $f^{\text {res }} \sim e^{2} a^{D-2}$. On the other hand, in order to provide the acceleration of a particle of mass $m$, one should apply the force $f=m a$. They become comparable $f^{\text {res }} \sim f$, when

$$
a_{*}^{D-3} \sim \frac{m}{e^{2}} .
$$

We denote by $l_{*}=a_{*}^{-1}$ a distance to the horizon corresponding to this solution. We also denote by $r_{m}$ a classical radius of the charged particle

$$
m=\frac{e^{2}}{r_{m}^{D-3}}
$$

then condition eq. (8.2) implies that

$$
l_{*} \sim r_{m}
$$

In other words the induced finite self-force correction, acting on the charge, is of the same order as the force, which produces the acceleration (directed towards the horizon [2]) when the distance to the horizon is small and comparable with the classical radius of the charge. One can expect, that study of such regimes would require more detailed knowledge concerning the internal structure of the particle, so that one cannot trust the obtained results in this range of parameters. In a way, this problem is similar to the famous problem of self-accelerated radiating charged particle motion in the classical electrodynamics (see, e.g. $[1,25,26])$.

As we have already told the static homogeneous gravitational field is an idealization. The corresponding Rindler metric naturally served as an approximation for the static metric of a compact object in a spacetime domain of the size small with respect to the size of the object (a black hole). An interesting question is how the expressions for the mass-shift due to the self-interaction for a particle in the homogeneous gravitational field and near the black hole are connected. In four-dimensional case this problem was analyzed long time ago (see, e.g., [4-6]). The self-energy shift for a charged particle near a black hole can be written in the form

$$
E^{\mathrm{res}}=\frac{1}{2} e^{2} a .
$$

This expression is similar to eq. (1.2), but differs from it by the sign. This difference is a result of two factors: (1) the topology of the black hole exterior differs from the topology of the Rindler space in the external with respect to the horizon domain; (2) the integral expression for the electromagnetic field energy is not uniformly convergent, so that its 
limit when $M \rightarrow \infty$ differs from a similar integral, in which this limit is performed 'inside' the integral. As a result of the non-trivial spatial topology of a black hole, the Maxwell equations for a static electric field in its background allow the existence of a 'zero-mode'. It describes a spherically symmetric electric field, which does not have sources in the black hole exterior. By adding such a solution one can make a black hole to be 'weakly charged'. For the self-mass problem one chooses the Green functions so that such a mode is suppressed and the black hole remains uncharged. Then the electric potential on the horizon, which is always constant, does not vanish. In the homogeneous gravitational field such zero-modes are absent and a regular solution has zero potential at the horizon. It is interesting, whether a similar phenomenon occurs in the higher dimensions.

As we mentioned, in the odd dimensional cases the expressions for the residual selfenergy and self-force contain logarithmic factors. Similar factors were discovered in [2] for the self-energy problem near 5-dimensional black hole. It is quite interesting question, what is a physical meaning of the cut-off parameter, that enters these expressions. One of the options is that it might be related with the scale that controls the validity of the homogeneous gravitational field approximation.

To obtain finite expressions for the self-energy and self-force we used special prescription for its regularization. This method is quite natural and basically it is some covariant version of the Hadamard regularization. However, an interesting question is whether there is an agreement in the results for different types of such 'covariant' regularizations. One certainly expects that the results obtained for a covariant regularization are more robust, than, say, for more naive regularizations, such as extended charged sphere. In the latter case an inevitable problem is connected with an ambiguity of the charge density distribution over the 'particle'. In higher dimensions this problem is more severe than in the 4-dimensional case.

In our approach we study a pure classical problem: the Planck constant does not enter any of the relations. However, it is quite interesting to consider a similar problem in the quantum field theory. In the four dimensional case Ritus $[27,28]$ calculated the shift of the self-mass for an electron moving in the static homogeneous electric field. His 'quantum' results were in good agreement with the classical calculations (for details, see [5, 6]). It is worthwhile to repeat similar comparison in higher dimensions.

\section{Acknowledgments}

The authors are grateful to Eric Poisson for inspiring discussions. The authors also are grateful to the referee for concrete and constructive suggestions of a better form of presenting the results. This work was partly supported by the Natural Sciences and Engineering Research Council of Canada. The authors are also grateful to the Killam Trust for its financial support.

\section{A Motion of a continuous charged medium in a curved spacetime}

\section{A.1 Fock's approach}

In the present paper we focus on the properties of point charges. However, it is instructive to consider this case as a special limit of continuous media, when its density takes the form 
of the delta-like distribution. Using this approach we derive in this appendix the equation of motion and the stress-energy tensor for charged media moving in a curved spacetime. For the electrically charged media we reproduce well known results. For the case of scalar charge we derive a consistent set of equations which have quite interesting form and allows us to analyze the motion of scalar charges in a curved spacetime in a self-consistent form. To derive of the equation of motion for distributions of electric or scalar charges we use $n$ approach developed by Fock [29] for the electrically charged media. We assume the flow lines of the media do not intersect, so that there are no caustics. In such a case the flow lines determine a spacetime foliation by 1-dimensional lines, which can be parametrized as follows

$$
X^{\alpha}=\chi^{\alpha}\left(\lambda, y^{b}\right)
$$

For fixed value of the parameters $y^{b}$, eq. (A.1) defines a single flow line with $\lambda$ as a 'time' parameter along it. We denote a partial derivative along the flow line with respect to $\lambda$ by a dot: $(\ldots)=\partial_{\lambda}(\ldots)$. The velocity of the motion of the element of the media along its flow line is

$$
u^{\alpha}=\dot{\chi}^{\alpha} / L, \quad L=\sqrt{-g_{\mu \nu} \dot{\chi}^{\mu} \dot{\chi}^{\nu}}, \quad u_{\alpha} u^{\alpha}=-1 .
$$

We assume that the proper mass $\mu(X)$, as well as the electric and scalar charge $\rho(X)$ and $J(X)$, within a given pencil of flow lines remain constant, so that the following continuity equations are automatically satisfied [29].

$$
\begin{aligned}
\nabla_{\alpha}\left(\mu u^{\alpha}\right) & =0, \\
\nabla_{\alpha}\left(J u^{\alpha}\right) & =0, \\
\nabla_{\alpha}\left(\rho u^{\alpha}\right) \equiv \nabla_{\alpha}\left(J^{\alpha}\right) & =0 .
\end{aligned}
$$

In the case of electric charges the conservation of the electric current $J^{\mu}$ also follows from the Maxwell equations.

Variation of the flow lines, which is the variation of the function $\chi^{\alpha}$, is described by displacement vector

$$
\eta^{\alpha}=\hat{\delta} X^{\alpha}=\hat{\delta} \chi^{\alpha}\left(\lambda, y^{b}\right)=\partial_{y^{b}} \chi^{\alpha}\left(\lambda, y^{b}\right) \delta y^{a}
$$

Consider the variation $\tilde{\delta}$ due to the change in the form of the functions $\chi^{\alpha}$.

$$
\begin{aligned}
u^{\mu}\left(X^{\alpha}+\eta^{\alpha}\right) & =u^{\mu}\left(X^{\alpha}\right)+\tilde{\delta} u^{\mu}, \\
\hat{\delta} u^{\mu} & =\tilde{\delta} u^{\mu}-\frac{\partial u^{\mu}}{\partial X^{\alpha}} \eta^{\alpha}
\end{aligned}
$$

Taking into account that

$$
\tilde{\delta} L=L^{-1}\left[\frac{1}{2} \frac{\partial g_{\mu \nu}^{\mathrm{D}}}{\partial X^{\sigma}} \eta^{\sigma} \dot{\chi}^{\mu} \dot{\chi}^{\nu}+g_{\mu \nu} \dot{\chi}^{\mu} \dot{\eta}^{\nu}\right]
$$

one can write

$$
\tilde{\delta} L=-L u_{\sigma} u^{\epsilon} \nabla_{\epsilon} \eta^{\sigma}
$$


Using these variation rules we obtain

$$
\tilde{\delta} u^{\alpha}=u^{\epsilon} \frac{\partial \eta^{\alpha}}{\partial X^{\epsilon}}+u^{\alpha} u_{\sigma} u^{\epsilon} \nabla_{\epsilon} \eta^{\sigma}
$$

and, hence,

$$
\hat{\delta} u^{\alpha}=u^{\epsilon} \nabla_{\epsilon} \eta^{\alpha}-\eta^{\epsilon} \nabla_{\epsilon} u^{\alpha}+u^{\alpha} u_{\sigma} u^{\epsilon} \nabla_{\epsilon} \eta^{\sigma}
$$

The variations of the mass $\mu(X)$ and scalar charge $J(X)$ densities are

$$
\begin{aligned}
& \frac{\tilde{\delta} \mu}{\mu}+\nabla_{\sigma} \eta^{\sigma}=-u_{\sigma} u^{\epsilon} \nabla_{\epsilon} \eta^{\sigma}, \\
& \frac{\tilde{\delta} J}{J}+\nabla_{\sigma} \eta^{\sigma}=-u_{\sigma} u^{\epsilon} \nabla_{\epsilon} \eta^{\sigma}, \\
& \frac{\tilde{\delta} \rho}{\rho}+\nabla_{\sigma} \eta^{\sigma}=-u_{\sigma} u^{\epsilon} \nabla_{\epsilon} \eta^{\sigma} .
\end{aligned}
$$

Finally the variation of the densities take a form

$$
\begin{aligned}
& \hat{\delta} \mu=-\nabla_{\alpha}\left(\mu \eta^{\alpha}\right)-\mu u_{\sigma} u^{\epsilon} \nabla_{\epsilon} \eta^{\sigma}, \\
& \hat{\delta} J=-\nabla_{\alpha}\left(J \eta^{\alpha}\right)-J u_{\sigma} u^{\epsilon} \nabla_{\epsilon} \eta^{\sigma}, \\
& \hat{\delta} \rho=-\nabla_{\alpha}\left(\rho \eta^{\alpha}\right)-\rho u_{\sigma} u^{\epsilon} \nabla_{\epsilon} \eta^{\sigma} .
\end{aligned}
$$

Here are the other useful variations

$$
\begin{aligned}
& \hat{\delta}\left(\mu u^{\alpha}\right)=\nabla_{\sigma}\left(\mu u^{\sigma} \eta^{\alpha}-\mu u^{\alpha} \eta^{\sigma}\right) \\
& \hat{\delta}\left(J u^{\alpha}\right)=\nabla_{\sigma}\left(J u^{\sigma} \eta^{\alpha}-J u^{\alpha} \eta^{\sigma}\right) \\
& \hat{\delta}\left(\rho u^{\alpha}\right)=\nabla_{\sigma}\left(\rho u^{\sigma} \eta^{\alpha}-\rho u^{\alpha} \eta^{\sigma}\right) .
\end{aligned}
$$

\section{A.2 Electrically charged media}

\section{A.2.1 Equations of motion}

Let us consider the flow of electrically charged particles with the current density $J^{\mu}(X)=$ $\rho(X) u^{\mu}$ and mass density $\mu(X)$. The action for the Maxwell field including an interaction term with the current of massive particles reads

$$
I=-\frac{1}{16 \pi} \int d X \sqrt{-g^{\mathrm{D}}} F^{\mu \nu} F_{\mu \nu}+\int d X \sqrt{-g^{\mathrm{D}}} A_{\mu} \rho u^{\mu}-\int d X \sqrt{-g^{\mathrm{D}}} \mu .
$$

Using the variational rule eq. (A.21) derived in this appendix, the continuity equation, and

$$
\hat{\delta} \int d X \sqrt{-g^{\mathrm{D}}} \mu=\int d X \sqrt{-g^{\mathrm{D}}} \hat{\delta} \mu-\int d X \sqrt{-g^{\mathrm{D}}} \mu w_{\sigma} \eta^{\sigma}
$$

we get

$$
\begin{aligned}
\hat{\delta} I= & -\frac{1}{4 \pi} \int d X \sqrt{-g^{\mathrm{D}}} \hat{\delta} A_{\mu}\left(F_{; \sigma}^{\mu \sigma}-4 \pi \rho u^{\mu}\right) \\
& -\int d X \sqrt{-g^{\mathrm{D}}} \eta^{\alpha}\left[\mu w_{\alpha}-\rho u^{\beta} F_{\alpha \beta}\right] .
\end{aligned}
$$


Here $w^{\alpha}$ is the acceleration vector

$$
w^{\alpha}=u^{\epsilon} \nabla_{\epsilon} u^{\alpha}
$$

Thus the equations of motion are

$$
\begin{aligned}
F_{; \sigma}^{\mu \sigma} & =4 \pi J^{\mu} & J^{\mu} & =\rho u^{\mu} . \\
\mu w_{\alpha} & =\mathrm{f}_{\alpha} & \mathrm{f}_{\alpha} & =F_{\alpha \beta} J^{\beta} .
\end{aligned}
$$

The vector $\mathrm{f}_{\alpha}$ defines the local force density acting on the element of an electrically charged medium.

\section{A.2.2 Stress-energy tensor}

We denote the variations over the metric using the symbol $\delta$

$$
\frac{\delta(\mu \sqrt{-g})}{\mu \sqrt{-g}}=\frac{\left(\partial \chi^{\mu} / \partial p\right)\left(\partial \chi^{\nu} / \partial p\right)}{2 g_{\alpha \beta}\left(\partial \chi^{\alpha} / \partial p\right)\left(\partial \chi^{\beta} / \partial p\right)} \delta g_{\mu \nu}=-\frac{1}{2} u^{\mu} u^{\nu} \delta g_{\mu \nu}
$$

The stress-energy tensor $T^{\mu \nu}=\frac{2}{\sqrt{-g}} \frac{\delta I}{\delta g_{\mu \nu}}$ reads

$$
T^{\mu \nu}=\frac{1}{4 \pi}\left(F^{\mu \sigma} F_{\sigma}^{\nu}-\frac{1}{4} g^{\mu \nu} F^{\alpha \beta} F_{\alpha \beta}\right)+u^{\mu} u^{\nu} \mu .
$$

Note that metric variations of the interaction term vanishes. The divergence of the stressenergy tensor

$$
T_{\alpha \beta} ; \beta=\mu w_{\alpha}-F_{\alpha \beta} J^{\beta}
$$

must vanish for a closed system. It does vanish on the equations of motion eq. (A.27).

\section{A.3 Motion of the media with the scalar charge}

\section{A.3.1 Equations of motion}

Let us consider the flow of scalar particles with charge distribution $J(X)$ and mass density $\mu(X)$ in a curved spacetime. A minimally coupled massless scalar field $\Phi(X)$ with is described by an action

$$
I=-\frac{1}{8 \pi} \int d X \sqrt{-g^{\mathrm{D}}} \Phi^{; \mu} \Phi_{; \mu}+\int d X \sqrt{-g^{\mathrm{D}}} J \Phi-\int d X \sqrt{-g^{\mathrm{D}}} \mu .
$$

Using the variational rules derived in this appendix and the continuity equations we get

$$
\begin{aligned}
\hat{\delta} \int d X \sqrt{-g^{\mathrm{D}}} \mu & =\int d X \sqrt{-g^{\mathrm{D}}} \hat{\delta} \mu=\int d X \sqrt{-g^{\mathrm{D}}} \mu w_{\sigma} \eta^{\sigma}, \\
\hat{\delta} \int d X \sqrt{-g^{\mathrm{D}}} J \Phi & =\int d X \sqrt{-g^{\mathrm{D}}} \Phi \hat{\delta} J \\
& =\int d X \sqrt{-g^{\mathrm{D}}} J \eta^{\sigma}\left(\Phi w_{\sigma}+\nabla_{\sigma} \Phi+u_{\sigma} u^{\epsilon} \nabla_{\epsilon} \Phi\right),
\end{aligned}
$$

where $w^{\alpha}$ is the acceleration vector

$$
w^{\alpha}=u^{\epsilon} \nabla_{\epsilon} u^{\alpha}
$$


The variation of the action then reads

$$
\begin{aligned}
\hat{\delta} I= & \frac{1}{4 \pi} \int d X \sqrt{-g^{\mathrm{D}}} \hat{\delta} \Phi\left(\Phi_{; \mu}^{; \mu}+4 \pi J\right) \\
& -\int d X \sqrt{-g^{\mathrm{D}}} \eta^{\sigma}\left[(\mu-\Phi J) w_{\sigma}-J\left(\nabla_{\sigma} \Phi+u_{\sigma} u^{\epsilon} \nabla_{\epsilon} \Phi\right)\right] .
\end{aligned}
$$

Thus the equation of motion are

$$
\begin{aligned}
\square \Phi & =-4 \pi J . \\
\mu w_{\alpha} & =\mathrm{f}_{\alpha}, \quad \mathrm{f}_{\alpha}=J\left(\delta_{\alpha}^{\beta}+u_{\alpha} u^{\beta}\right) \nabla_{\beta} \Phi+w_{\alpha} \Phi J .
\end{aligned}
$$

The vector $\mathrm{f}_{\alpha}$ defines the local force density acting on the element of a charged medium.

\section{A.3.2 Stress-energy tensor}

We denote the variations over the metric using the symbol $\delta$.

$$
\frac{\delta(\mu \sqrt{-g})}{\mu \sqrt{-g}}=\frac{\left(\partial \chi^{\mu} / \partial p\right)\left(\partial \chi^{\nu} / \partial p\right)}{2 g_{\alpha \beta}\left(\partial \chi^{\alpha} / \partial p\right)\left(\partial \chi^{\beta} / \partial p\right)} \delta g_{\mu \nu}=-\frac{1}{2} u^{\mu} u^{\nu} \delta g_{\mu \nu}
$$

The stress-energy tensor $T^{\mu \nu}=\frac{2}{\sqrt{-g}} \frac{\delta I}{\delta g_{\mu \nu}}$ is given by the expression

$$
T^{\mu \nu}=\frac{1}{4 \pi}\left(\Phi^{; \mu} \Phi^{; \nu}-\frac{1}{2} g^{\mu \nu} \Phi^{; \alpha} \Phi_{; \alpha}\right)+u^{\mu} u^{\nu}(\mu-\Phi J) .
$$

Note that, in contrast to the Maxwell field, it contains the term $u^{\mu} u^{\nu} \Phi J$ explicitly depending on the charge density $J$. The divergence of the stress-energy tensor

$$
T_{\alpha \beta} ; \beta=(\mu-\Phi J) w_{\alpha}-J\left(\delta_{\alpha}^{\beta}+u_{\alpha} u^{\beta}\right) \nabla_{\beta} \Phi
$$

must vanish for a closed system. Thus, we re-derive by a different method the motion equations eq. (A.37) for the medium.

\section{B Green Functions}

The regularized static Green functions, both for scalar and electromagnetic fields, are given by

$$
G_{\epsilon}=\frac{\sqrt{z z^{\prime}}}{2 \pi^{\beta}} I_{\nu}^{\beta}, \quad \beta=\frac{D}{2}-1 .
$$

The parameter $\nu=0$ for the scalar field and $\nu=1$ for the Maxwell field.

$$
\begin{aligned}
I_{\nu}^{\eta} & =\int_{0}^{\infty} u^{\eta-1} e^{-p u} I_{\nu}(c u) d u \\
& =p^{-\eta-\nu}\left(\frac{c}{2}\right)^{\nu} \frac{\Gamma(\nu+\eta)}{\Gamma(\nu+1)} F\left(\frac{\eta+\nu}{2}, \frac{\eta+\nu+1}{2} ; \nu+1 ; \frac{c^{2}}{p^{2}}\right) \\
& =e^{-\pi \nu i / 2} \Gamma(\eta+\nu)\left(p^{2}-c^{2}\right)^{-\eta / 2} P_{\eta-1}^{-\nu}\left(\frac{p}{\sqrt{p^{2}-c^{2}}}\right)
\end{aligned}
$$


where

$$
u=\frac{1}{4 s}, \quad p=z^{2}+z^{\prime 2}+x_{\perp}^{2}+\epsilon^{2}, \quad c=2 z z^{\prime},
$$

and

$$
\Re(\eta)>0, \quad \Re(p)>|\Re(c)| .
$$

For particular dimensions the integrals $I_{\nu}^{\eta}$ can be written in terms of elliptic functions. Let

$$
k=\sqrt{\frac{2 c}{p+c}},
$$

then

$$
\begin{aligned}
I_{0}^{1 / 2} & =k \sqrt{\frac{2}{\pi c}} \mathbf{K}(k) \\
I_{0}^{1} & =\frac{1}{\left(p^{2}-c^{2}\right)^{1 / 2}}, \\
I_{0}^{3 / 2} & =\frac{k^{3}}{2\left(1-k^{2}\right) \sqrt{2 \pi c^{3}}} \mathbf{E}(k), \\
I_{0}^{2} & =\frac{p}{\left(p^{2}-c^{2}\right)^{3 / 2}}, \\
I_{0}^{5 / 2} & =\frac{k^{5}}{8\left(1-k^{2}\right)^{2} \sqrt{2 \pi c^{5}}}\left[2\left(2-k^{2}\right) \mathbf{E}(k)-\left(1-k^{2}\right) \mathbf{K}(k)\right], \\
I_{0}^{3} & =\frac{2 p^{2}+c^{2}}{\left(p^{2}-c^{2}\right)^{5 / 2}}, \\
I_{1}^{1 / 2} & =\frac{1}{k} \sqrt{\frac{2}{\pi c}}\left[\left(2-k^{2}\right) \mathbf{K}(k)-2 \mathbf{E}(k)\right] \\
I_{1}^{1} & =\frac{c}{\left(p^{2}-c^{2}\right)^{1 / 2}\left(p+\left(p^{2}-c^{2}\right)^{1 / 2}\right)}, \\
I_{1}^{3 / 2} & =\frac{k}{2\left(1-k^{2}\right) \sqrt{2 \pi c^{3}}}\left[\left(2-k^{2}\right) \mathbf{E}(k)-2\left(1-k^{2}\right) \mathbf{K}(k)\right] \\
I_{1}^{2} & =\frac{c}{\left(p^{2}-c^{2}\right)^{3 / 2}}, \\
I_{1}^{5 / 2} & =\frac{k^{3}}{8\left(1-k^{2}\right)^{2} \sqrt{2 \pi c^{5}}}\left[2\left(1-k^{2}+k^{4}\right) \mathbf{E}(k)-\left(1-k^{2}\right)\left(2-k^{2}\right) \mathbf{K}(k)\right], \\
I_{1}^{3} & =\frac{3 p c}{\left(p^{2}-c^{2}\right)^{5 / 2}} \cdot
\end{aligned}
$$

\section{Divergences and finite parts of the Green functions}

\section{C.1 Maxwell field}

In three dimensions we get

$$
\begin{aligned}
G_{\epsilon}^{(3)} & =-\frac{1}{2 \pi}\left[\ln \left(\frac{\epsilon}{8 z}\right)+2\right]+O\left(\epsilon^{2}\right), \\
G^{(3) \mathrm{div}} & =-\frac{1}{2 \pi}[\ln (\epsilon \lambda)-\ln 2+\gamma], \\
G^{(3) \mathrm{ren}} & =\frac{1}{2 \pi}[\ln (\lambda z)+2 \ln 2+\gamma-2] .
\end{aligned}
$$


In four dimensions

$$
\begin{aligned}
G_{\epsilon}^{(4)} & =\frac{1}{4 \pi \epsilon}-\frac{1}{4 \pi z}+O(\epsilon), \\
G^{(4) \mathrm{div}} & =\frac{1}{4 \pi} \frac{1}{\epsilon}, \\
G^{(4) \mathrm{ren}} & =-\frac{1}{4 \pi z} .
\end{aligned}
$$

In five dimensions

$$
\begin{aligned}
G_{\epsilon}^{(5)} & =\frac{1}{4 \pi^{2}}\left[\frac{1}{\epsilon^{2}}+\frac{3}{8 z^{2}}\left(\ln \left(\frac{\epsilon}{8 z}\right)+\frac{5}{6}\right)\right]+O\left(\epsilon^{2}\right), \\
G^{(5) \text { div }} & =\frac{1}{4 \pi^{2}}\left[\frac{1}{\epsilon^{2}}+\frac{3}{8 z^{2}}(\ln (\epsilon \lambda)-\ln 2+\gamma)\right], \\
G^{(5) \text { ren }} & =-\frac{3}{32 \pi^{2} z^{2}}[\ln (\lambda z)+2 \ln 2+\gamma-5 / 6] .
\end{aligned}
$$

In six dimensions

$$
\begin{aligned}
G_{\epsilon}^{(6)} & =\frac{1}{8 \pi^{2} \epsilon^{3}}-\frac{3}{64 \pi^{2} z^{2} \epsilon}+O(\epsilon), \\
G^{(6) \mathrm{div}} & =\frac{1}{8 \pi^{2} \epsilon^{3}}-\frac{3}{64 \pi^{2} z^{2} \epsilon}, \\
G^{(6) \mathrm{ren}} & =0 .
\end{aligned}
$$

In seven dimensions

$$
\begin{aligned}
G_{\epsilon}^{(7)} & =\frac{1}{4 \pi^{3}}\left[\frac{1}{\epsilon^{4}}-\frac{3}{16 z^{2} \epsilon^{2}}+\frac{15}{256 z^{4}}\left(\ln \left(\frac{\epsilon}{8 z}\right)+\frac{107}{60}\right)\right]+O\left(\epsilon^{2}\right), \\
G^{(7) \mathrm{div}} & =\frac{1}{4 \pi^{3}}\left[\frac{1}{\epsilon^{4}}-\frac{3}{16 z^{2} \epsilon^{2}}+\frac{15}{256 z^{4}}(\ln (\epsilon \lambda)-\ln 2+\gamma)\right], \\
G^{(7) \mathrm{ren}} & =-\frac{15}{1024 \pi^{3} z^{4}}[\ln (\lambda z)+2 \ln 2+\gamma-107 / 60] .
\end{aligned}
$$

In eight dimensions

$$
\begin{aligned}
G_{\epsilon}^{(8)} & =\frac{3}{16 \pi^{3}}\left[\frac{1}{\epsilon^{5}}-\frac{1}{8 z^{2} \epsilon^{3}}-\frac{5}{128 z^{4} \epsilon}\right]+O(\epsilon), \\
G^{(8) \text { div }} & =\frac{3}{16 \pi^{3}}\left[\frac{1}{\epsilon^{5}}-\frac{1}{8 z^{2} \epsilon^{3}}-\frac{5}{128 z^{4} \epsilon}\right], \\
G^{(8)_{\text {ren }}} & =0 .
\end{aligned}
$$

\section{C.2 Scalar field}

In three dimensions we get

$$
\begin{aligned}
G_{\epsilon}^{(3)} & =-\frac{1}{2 \pi} \ln \left(\frac{\epsilon}{8 z}\right)+O\left(\epsilon^{2}\right), \\
G^{(3) \mathrm{div}} & =-\frac{1}{2 \pi}[\ln (\epsilon \lambda)-\ln 2+\gamma], \\
G^{(3) \mathrm{ren}} & =\frac{1}{2 \pi}[\ln (\lambda z)+2 \ln 2+\gamma] .
\end{aligned}
$$


In four dimensions

$$
\begin{aligned}
G_{\epsilon}^{(4)} & =\frac{1}{4 \pi \epsilon}+O(\epsilon), \\
G^{(4) \text { div }} & =\frac{1}{4 \pi \epsilon}, \\
G^{(4) \text { ren }} & =0 .
\end{aligned}
$$

In five dimensions

$$
\begin{aligned}
G_{\epsilon}^{(5)} & =\frac{1}{4 \pi^{2}}\left[\frac{1}{\epsilon^{2}}-\frac{1}{8 z^{2}}\left(\ln \left(\frac{\epsilon}{8 z}\right)+\frac{3}{2}\right)\right]+O\left(\epsilon^{2}\right), \\
G^{(5) \text { div }} & =\frac{1}{4 \pi^{2}}\left[\frac{1}{\epsilon^{2}}-\frac{1}{8 z^{2}}(\ln (\epsilon \lambda)-\ln 2+\gamma)\right], \\
G^{(5) \text { ren }} & =\frac{1}{32 \pi^{2} z^{2}}[\ln (\lambda z)+2 \ln 2+\gamma-3 / 2] .
\end{aligned}
$$

In six dimensions

$$
\begin{aligned}
G_{\epsilon}^{(6)} & =\frac{1}{8 \pi^{2}}\left[\frac{1}{\epsilon^{3}}+\frac{1}{8 z^{2} \epsilon}\right]+O(\epsilon), \\
G^{(6)_{\text {div }}} & =\frac{1}{8 \pi^{2}}\left[\frac{1}{\epsilon^{3}}+\frac{1}{8 z^{2} \epsilon}\right], \\
G^{(6)_{\text {ren }}} & =0 .
\end{aligned}
$$

In seven dimensions

$$
\begin{aligned}
G_{\epsilon}^{(7)} & =\frac{1}{4 \pi^{3}}\left[\frac{1}{\epsilon^{4}}+\frac{1}{16 z^{2} \epsilon^{2}}-\frac{9}{256 z^{4}}\left(\ln \left(\frac{\epsilon}{8 z}\right)+\frac{23}{12}\right)\right]+O\left(\epsilon^{2}\right), \\
G^{(7) \mathrm{div}} & =\frac{1}{4 \pi^{3}}\left[\frac{1}{\epsilon^{4}}+\frac{1}{16 z^{2} \epsilon^{2}}-\frac{9}{256 z^{4}}(\ln (\epsilon \lambda)-\ln 2+\gamma)\right], \\
G^{(7) \mathrm{ren}} & =\frac{9}{1024 \pi^{3} z^{4}}[\ln (\lambda z)+2 \ln 2+\gamma-23 / 12] .
\end{aligned}
$$

In eight dimensions

$$
\begin{aligned}
G_{\epsilon}^{(8)} & =\frac{3}{16 \pi^{3} \epsilon^{5}}+\frac{1}{128 \pi^{3} z^{2} \epsilon^{3}}+\frac{9}{2048 \pi^{3} z^{4} \epsilon}+O(\epsilon), \\
G^{(8) \text { div }} & =\frac{3}{16 \pi^{3} \epsilon^{5}}+\frac{1}{128 \pi^{3} z^{2} \epsilon^{3}}+\frac{9}{2048 \pi^{3} z^{4} \epsilon}, \\
G^{(8) \text { ren }} & =0 .
\end{aligned}
$$

Open Access. This article is distributed under the terms of the Creative Commons Attribution License (CC-BY 4.0), which permits any use, distribution and reproduction in any medium, provided the original author(s) and source are credited.

\section{References}

[1] L. Landau and E. Lifschits, The Classical Theory of Fields, Pergamon Press (1982). 
[2] M.J.S. Beach, E. Poisson and B. G. Nickel, Self-force on a charge outside a five-dimensional black hole, Phys. Rev. D 89 (2014) 124014.

[3] E. Fermi, Sull'elettrostatica di un campo gravitazionale uniforme e sul peso delle masse elettromagnetiche, Nuovo Cim. 22 (1921) 176.

[4] A.G. Smith and C.M. Will, Force on a static charge outside a Schwarzschild black hole, Phys. Rev. D 22 (1980) 1276 [InSPIRE].

[5] A. Zelnikov and V. Frolov, Influence of gravitation on the self-energy of charged particles, Sov. Phys. JETP 55 (1982) 191.

[6] A. Zelnikov and V. P. Frolov, The influence of gravitation, acceleration, and temperature on the self-energy of charged particles (in Russian), Proc. Lebedev Phys. Inst. 152 (1983) 96.

[7] L.M. Burko and Y.T. Liu, Selfforce on a scalar charge in the space-time of a stationary, axisymmetric black hole, Phys. Rev. D 64 (2001) 024006 [gr-qc/0103008] [INSPIRE].

[8] V.P. Frolov and A. Zelnikov, Self-energy of a scalar charge near higher-dimensional black holes, Phys. Rev. D 85 (2012) 124042 [arXiv:1204.3122] [INSPIRE].

[9] V.P. Frolov and A. Zelnikov, Classical self-energy and anomaly, Phys. Rev. D 86 (2012) 044022 [arXiv: 1205.4269] [inSPIRE].

[10] V.P. Frolov and A. Zelnikov, Anomaly and the self-energy of electric charges, Phys. Rev. D 86 (2012) 104021 [arXiv: 1208.5763] [INSPIRE].

[11] V.P. Frolov, A.A. Shoom and A. Zelnikov, Self-energy anomaly of an electric pointlike dipole in three-dimensional static spacetimes, Phys. Rev. D 88 (2013) 024032 [arXiv:1303.1816] [INSPIRE].

[12] D.V. Gal'tsov and P. Spirin, Radiation reaction reexamined: Bound momentum and Schott term, Grav. Cosmol. 12 (2006) 1 [hep-th/0405121] [INSPIRE].

[13] D.V. Gal'tsov and P.A. Spirin, Radiation reaction in curved even-dimensional spacetime, Grav. Cosmol. 13 (2007) 241 [arXiv: 1012. 3085] [INSPIRE].

[14] B.P. Kosyakov, Exact solutions of classical electrodynamics and the Yang-Mills-Wong theory in even-dimensional space-time, Theor. Math. Phys. 119 (1999) 493 [hep-th/0207217] [INSPIRE].

[15] S. Isoyama and E. Poisson, Self-force as probe of internal structure, Class. Quant. Grav. 29 (2012) 155012 [arXiv:1205.1236] [inSPIRE].

[16] B.S. DeWitt and R.W. Brehme, Radiation damping in a gravitational field, Annals Phys. 9 (1960) 220 [INSPIRE].

[17] E. Poisson, A. Pound and I. Vega, The Motion of point particles in curved spacetime, Living Rev. Rel. 14 (2011) 7 [arXiv:1102.0529] [INSPIRE].

[18] M. Casals, E. Poisson and I. Vega, Regularization of static self-forces, Phys. Rev. D 86 (2012) 064033 [arXiv:1206.3772] [INSPIRE].

[19] P. Zimmerman and E. Poisson, Gravitational self-force in nonvacuum spacetimes, arXiv: 1406.5111 [INSPIRE].

[20] T.C. Quinn and R.M. Wald, An Axiomatic approach to electromagnetic and gravitational radiation reaction of particles in curved space-time, Phys. Rev. D 56 (1997) 3381 [gr-qc/9610053] [INSPIRE]. 
[21] E.T. Copson, On electrostatics in a gravitational field, Proc. Roy. Soc. Lond. A 118 (1928) 184.

[22] B. Linet, Electrostatics and magnetostatics in the Schwarzschild metric, J. Phys. A 9 (1976) 1081 [INSPIRE].

[23] T.C. Quinn, Axiomatic approach to radiation reaction of scalar point particles in curved space-time, Phys. Rev. D 62 (2000) 064029 [gr-qc/0005030] [INSPIRE].

[24] V. Ginzburg, Physics and astrophysics: a selection of key problems, Pergamon Press (1985).

[25] F. Rohrlich, Dynamics of a charged particle, Phys. Rev. E 77 (2008) 046609.

[26] S.E. Gralla, A.I. Harte and R.M. Wald, A Rigorous Derivation of Electromagnetic Self-force, Phys. Rev. D 80 (2009) 024031 [arXiv:0905.2391] [InSPIRE].

[27] V.I. Ritus, Method of eigenfunctions and mass operator in quantum electrodynamics of a constant field, Sov. Phys. JETP 48 (1978) 788 [INSPIRE].

[28] V.I. Ritus, The Mass Shift of Accelerated Charge, Sov. Phys. JETP 53 (1981) 659 [InSPIRE].

[29] V. Fock, The theory of space, time and gravitation, translated by N. Kemmer, Pergamon Press (1964). 